\title{
The Gulf Stream North Wall: Ageostrophic Circulation and Frontogenesis
}

\author{
JAMES C. MCWILLIAMS \\ Department of Atmospheric and Oceanic Sciences, University of California, Los Angeles, Los Angeles, California \\ JONATHAN GULA \\ Univ. Brest, CNRS, IRD, Ifremer, Laboratoire d'Océanographie Physique et Spatiale, IUEM, Brest, France

\section{JEROEN MOLEMAKER} \\ Department of Atmospheric and Oceanic Sciences, University of California, Los Angeles, Los Angeles, California
}

(Manuscript received 28 September 2018, in final form 17 January 2019)

\begin{abstract}
Eastward zonal jets are common in the ocean and atmosphere, for example, the Gulf Stream and jet stream. They are characterized by atypically strong horizontal velocity, baroclinic vertical structure with an upward flow intensification, large change in the density stratification meridionally across the jet, largescale meanders around a central latitude, narrow troughs and broad crests, and a sharp and vertically sloping northern (poleward) "wall" defined by horizontal maxima in the lateral gradients of both velocity and density. Measurements and realistic oceanic simulations show these features in the Gulf Stream downstream from its western boundary separation point. A diagnostic theory based on the conservative balance equations is developed to calculate the 3D velocity field associated with the dynamic height field. When applied to an idealized representation of a meandering jet, it explains the spatial structure of the associated ageostrophic secondary circulation around the jet and the positive frontogenetic tendency along the northern wall in the meander sector located upstream from the trough. This provides a basis for understanding why submesoscale instabilities and cross-wall intrusion and streamer events are more prevalent along the sector downstream from the trough and at the crest where there is not such a frontogenetic tendency. An important attribute for this frontogenesis pattern is the 3D shape of the jet, whose idealization is summarized above.
\end{abstract}

\section{Introduction}

Strong, narrow eastward jets in the ocean and atmosphere often exhibit meanders in their path. Examples are the upper-ocean Gulf Stream, after separation from the western boundary at Cape Hatteras and before encountering the New England Seamount chain, and the midlatitude wintertime tropospheric jet stream. Although occasionally these meanders amplify to the point of pinching off isolated vortices (e.g., rings and cutoff lows, respectively), often they exhibit downstream propagation in a quasi-stable pattern for a period of days or longer. In such a regime a striking attribute of the jet shape is a sharp cross-jet front on its polar side in both density and horizontal velocity. The frontal width is

\footnotetext{
Corresponding author: James C. McWilliams, jcm@atmos.ucla. edu
}

observed to be quite narrow compared to the overall width of the high-speed flow region in the jet, often less than $5 \mathrm{~km}$ in the Gulf Stream and Kuroshio (Todd et al. 2016; Klymak et al. 2016; Nagai et al. 2009) and less than $100 \mathrm{~km}$ in the jet stream (Keyser and Shapiro 1986). These polar frontal widths are also much smaller than the length scales of the more general variability associated with the oceanic mesoscale $\left(\approx 10^{2} \mathrm{~km}\right)$ and atmospheric synoptic scale $\left(\approx 10^{3} \mathrm{~km}\right)$ flows engendered by the jet instabilities. Why?

The thesis in this paper is that the sharpness of the polar wall is a result of frontogenesis caused by the ageostrophic secondary circulation (i.e., flow in the plane perpendicular to the main geostrophic jet axis) that arises from a combination of the meandering jet path and several characteristic asymmetries in the jet shape, namely, the relative narrowness of its trough and polar side, compared to the crest and equatorward side, and a downward 
and equatorward tilt of the horizontal density front underneath its level of maximum speed (i.e., at the surface in ocean and at the tropopause in the atmosphere). ${ }^{1}$ Also, the ageostrophic velocity is not very small compared to the geostrophic one for a strong jet, especially as part of the secondary circulation that is an essential component of the frontogenesis process. A particular demonstration of this thesis is made for the Gulf Stream based on high-resolution simulations and a diagnostic analysis of its secondary circulation and frontogenetic tendency (abbreviated as SCFT), but these attributes seem similarly applicable to other eastward, meandering jets, including the jet stream. A compounding feature more specific to subtropical oceanic gyre jets is a core region of especially warm surface water in winter carried poleward along the western boundary from lower latitudes and out into the separated, eastward jet region.

The theoretical framework for the diagnostic SCFT analysis is an assumption of approximate force balance, defined by the relative smallness of the acceleration, especially of the horizontally divergent component. Because the Rossby number (Ro $=V / f l$, with $V$ a downstream velocity, $l$ a cross-stream length, and $f$ the Coriolis frequency) is not small, a quasigeostrophic model (based on Ro $\rightarrow 0$ asymptotics) is insufficiently accurate, so a higher-order balance equations (BE) model is adopted. While force-balanced dynamics is incomplete compared to nature and realistic simulations (e.g., by its lack of inertial and internal gravity waves), it is nevertheless a relevant approximation that can reproduce many features of strong jets, especially if used in only a diagnostic mode rather than for a sustained prognostic integration with $\mathrm{Ro} \sim 1$ and broadband variability.

A meandering jet is a coherent structure in geophysical fluid dynamics in the sense that it recurrently manifests a distinctive spatial pattern and evolutionary sequence (life cycle) that persists longer than might be expected from an advective eddy turnover time $l / V$ that is a typical correlation time in a turbulent flow. As such, it is of particular interest to explain the behavior of a meandering jet in an idealized context, here by making particular assumptions about the shape of its density field, neglecting nonconservative mixing effects and interactions with other neighboring flows, and calculating the velocity field and frontogenetic tendency using the BE model. The goal is to achieve a basic understanding of an essentially universal phenomenon.

\footnotetext{
${ }^{1}$ Consistent with common meteorological usage, trough refers to the location of the part of the eastward meandering jet closest to the equation, and the crest is the part closest to the pole.
}

A similar SCFT diagnostic approach was taken to interpret another type of coherent structure, that is, oceanic submesoscale surface fronts and density filaments under the influence of vertical mixing in the turbulent boundary layer and Stokes drift from surface gravity waves (McWilliams 2017, 2018). Here the focus is instead on an essentially mesoscale jet; the turbulent mixing and surface gravity wave influences are neglected, and the choice of a force-balanced approximation is different (as explained in section 4).

In addition to the measurements presented in section 2, the present study is enabled by realistic, high-resolution, nested-grid simulations of the Gulf Stream in the offshore meandering region described in section 3. The BE-SCFT model is presented in section 4 , and an idealized shape for the density field of a meandering jet is specified in section 5. The BE-SCFT solutions are analyzed and compared with the simulation model's secondary circulation and frontogenetic tendencies in section 6 . The results are summarized and further discussed in section 7 .

\section{Observations of the meandering Gulf Stream}

The Gulf Stream is observed at high spatial resolution in sea surface temperature (SST) and ocean color satellite images (Fig. 1). The sharpness of the front at the North Wall of the Gulf Stream is evident starting near the separation point at Cape Hatteras $\left(35.3^{\circ} \mathrm{N}, 75.5^{\circ} \mathrm{W}\right)$. Downstream from separation, the Gulf Stream routinely forms large (mesoscale) meanders and eddies, but the North Wall's frontal width stays very narrow compared to the overall width of the jet, extending even hundreds of kilometers downstream from separation.

Frontal widths on the order of $5-10 \mathrm{~km}$ have been measured during the LatMix experiment at $60^{\circ}-65^{\circ} \mathrm{W}$ (Klymak et al. 2016) and from glider sections at $70^{\circ} \mathrm{W}$ (Todd et al. 2016). A typical transect across the Gulf Stream from an underwater glider around $62^{\circ}-65^{\circ} \mathrm{W}$ is shown in Fig. 2. It is extracted from the dataset of Todd and Owens (2016), which provides sections of temperature and salinity down to $1000-\mathrm{m}$ depth, with a resolution of approximately $5 \mathrm{~km}$ horizontally and $5.5 \mathrm{~h}$ in time (Todd et al. 2016). Considering that gliders were instructed to fly at right angles to the measured flow with velocities $\approx 0.25 \mathrm{~m} \mathrm{~s}^{-1}$ (Todd et al. 2016), we compute an approximate cross-stream distance $y^{\prime}$. The frontal width at the surface is on the order of $10 \mathrm{~km}$, with a horizontal gradient of buoyancy (i.e., $b=-g \rho / \rho_{0}$, where $g$ is gravity, $\rho$ is potential density, and $\rho_{0}$ is a reference value, $1025 \mathrm{~kg} \mathrm{~m}^{-3}$ ), on the order of $10^{-6} \mathrm{~s}^{-2}$. The horizontal buoyancy gradients (and associated geostrophic velocities) are maximum at the surface. A shallow, buoyant 

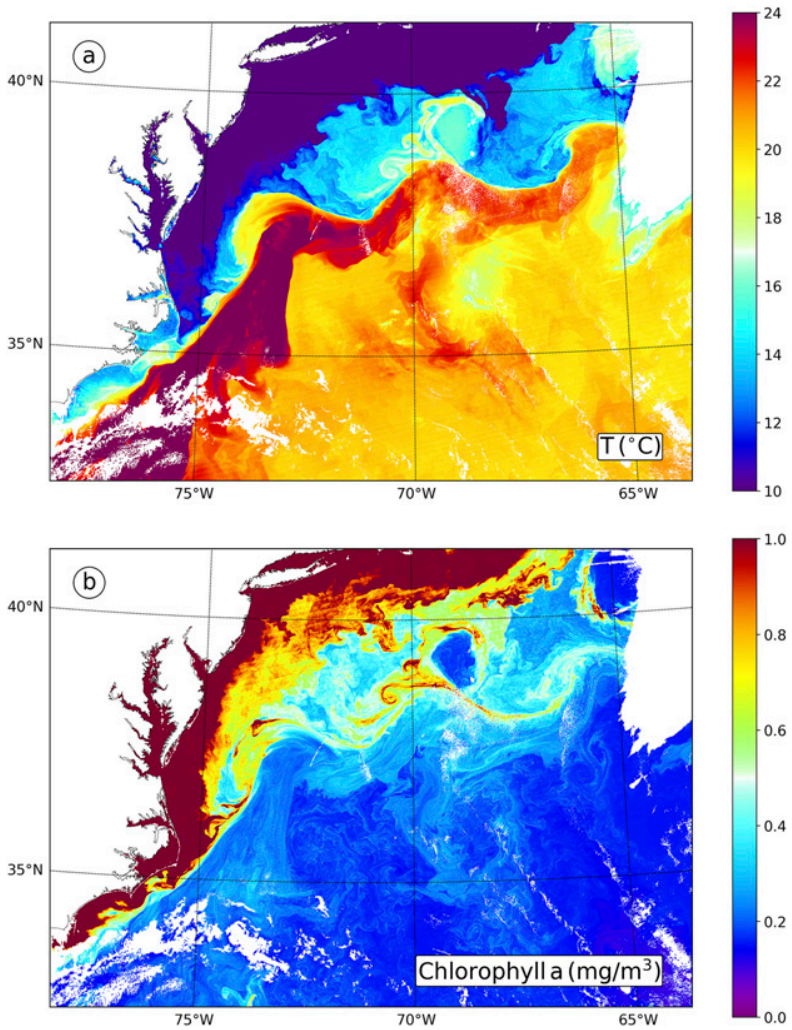

FIG. 1. (a) SST and (b) chlorophyll A concentration for the Gulf Stream on 9 Mar 2016, collected by the Visible Infrared Imaging Radiometer Suite on the NASA-NOAA Suomi-NPP satellite. Notice the smoother, narrower, less fragmented shape of the uptrough sectors and troughs compared to the downtrough sectors and crests.

surface-layer core, specific to subtropical oceanic gyre jets, extends south of the North Wall and forms a secondary frontal zone of weaker amplitude (i.e., the south wall). Below the surface the horizontal front is tilted equatorward with increasing depth, as observed for the tropospheric jet stream (Keyser and Shapiro 1986; Davies and Rossa 1998).

The sharpness of the front is also modulated depending on the phase of the meander (Fig. 1). The front is usually sharper in the meander sector upstream of the trough (hereafter abbreviated as uptrough) and at the trough rather than downstream of the trough (i.e., downtrough) and at the crest. The front appears looser and more disrupted by cross-front perturbations in the downtrough sectors (Fig. 1). Cross-front perturbations are visible in the Gulf Stream as submesoscale cold intrusions in the downtrough sectors of the North Wall in Fig. 1 or in a similar SST image in Klymak et al. (2016). They are also visible north of the Gulf Stream in the form of streamers of warm and salty water that detach from the core of the Gulf Stream usually in this sector (Klymak et al. 2016).

\section{Simulations of the meandering Gulf Stream}

Realistic simulations are made for the Gulf Stream in the offshore region after its western-boundary separation at Cape Hatteras.

\section{a. Model setup}

The Regional Oceanic Modeling System (ROMS; Shchepetkin and McWilliams 2005) is used. It is a code based on the hydrostatic primitive equations (PEs) with a free-surface- and terrain-following vertical coordinate; it includes surface wind and buoyancy forcing, open lateral boundary conditions, parameterized mixing in the vertical boundary layers, and a small-scale, dissipative regularization in the interior.

We use a nesting approach with successive horizontal grid nesting refinements from a parent grid resolution of $\Delta x \approx 6 \mathrm{~km}$, covering most of the Atlantic Ocean, to successive child grids with horizontal resolutions $\Delta x \approx 1.5 \mathrm{~km}$ and $\Delta x \approx 500 \mathrm{~m}$ over the Gulf Stream region (Fig. 3). The configuration of the Atlantic domain and the larger nest are described in Gula et al. (2014), and the finer nest is described in Klymak et al. (2016). The finer-resolution nest has $2000 \times 1600$ grid points with $\Delta x \approx 500 \mathrm{~m}$ in the horizontal and 50 vertical levels, covering a region of the Gulf Stream downstream from its separation from the U.S. continental slope at Cape Hatteras. All forcings and physical options are identical to the ones described in Gula et al. (2015), including daily winds and diurnally modulated surface buoyancy fluxes. Almost all the ROMS results presented here come from the finer-resolution nest (only Figs. 3 and 21 involve the coarser parent grids).

\section{b. Jet structure and secondary circulation}

The sea surface height ( $\mathrm{SSH}$ ) and surface velocity associated with a typical meandering state of the Gulf Stream are shown in Fig. 4a. They show, in particular, the two features already observed in satellite images (Fig. 1): a narrower jet on the polar side than the equatorial side (i.e., a sharp North Wall) and a narrower jet width in troughs than in crests.

Vertical sections of buoyancy and along-stream velocity, averaged along-stream in two sectors corresponding to a trough and a crest, are shown in Figs. $4 \mathrm{c}$ and $4 \mathrm{~d}$. The velocity of the jet is surface intensified, with stronger velocity amplitude and tighter horizontal buoyancy gradient in the trough, where the jet is narrower, compared to the crest. The vertical structure of buoyancy also shows the same two features observed in in situ hydrographic sections (Fig. 2): a buoyant surface-layer jet core and an equatorward tilt of the horizontal front with increasing depth. 

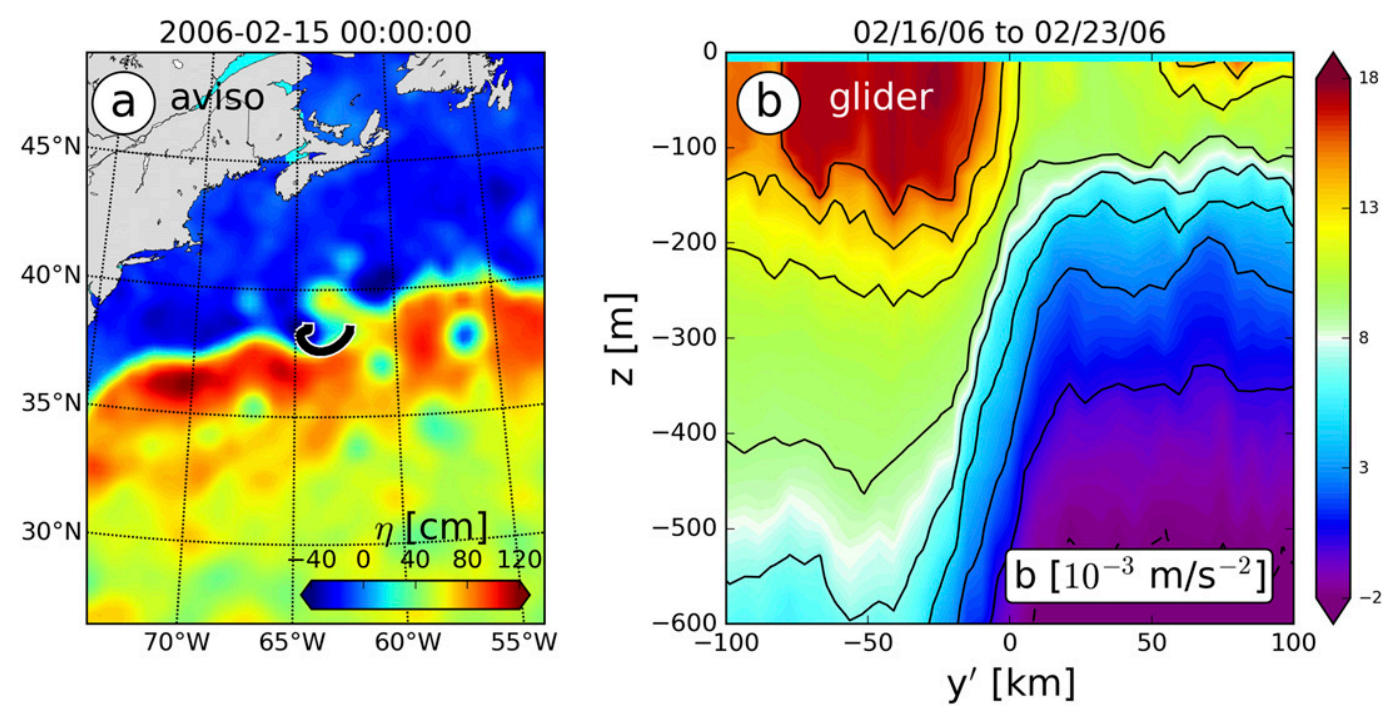

FIG. 2. (a) SSH (cm) from AVISO (Ducet et al. 2000) on 15 Feb 2006, and (b) buoyancy ( $\mathrm{m} \mathrm{s}^{-2}$ ) along a glider section taken between 16 and 23 Feb 2006, across the Gulf Stream North Wall. The glider trajectory is shown as black dots in (a).

The structure of vertical velocity $w$ and horizontal divergence $\delta=\nabla^{2} \chi$ [with $\nabla$ the horizontal gradient operator and $\chi$ the divergent potential; see (2)] in the Gulf Stream, as in other oceanic and atmospheric jets, is tightly linked to the meandering pattern of the jet
(Bower 1989). Measurements by Lindstrom et al. (1997) in the Gulf Stream show that vertical velocity patterns below the mixed layer are nearly phase-locked with meanders and tend to be downward/upward when entering/exiting troughs, with the largest amplitudes

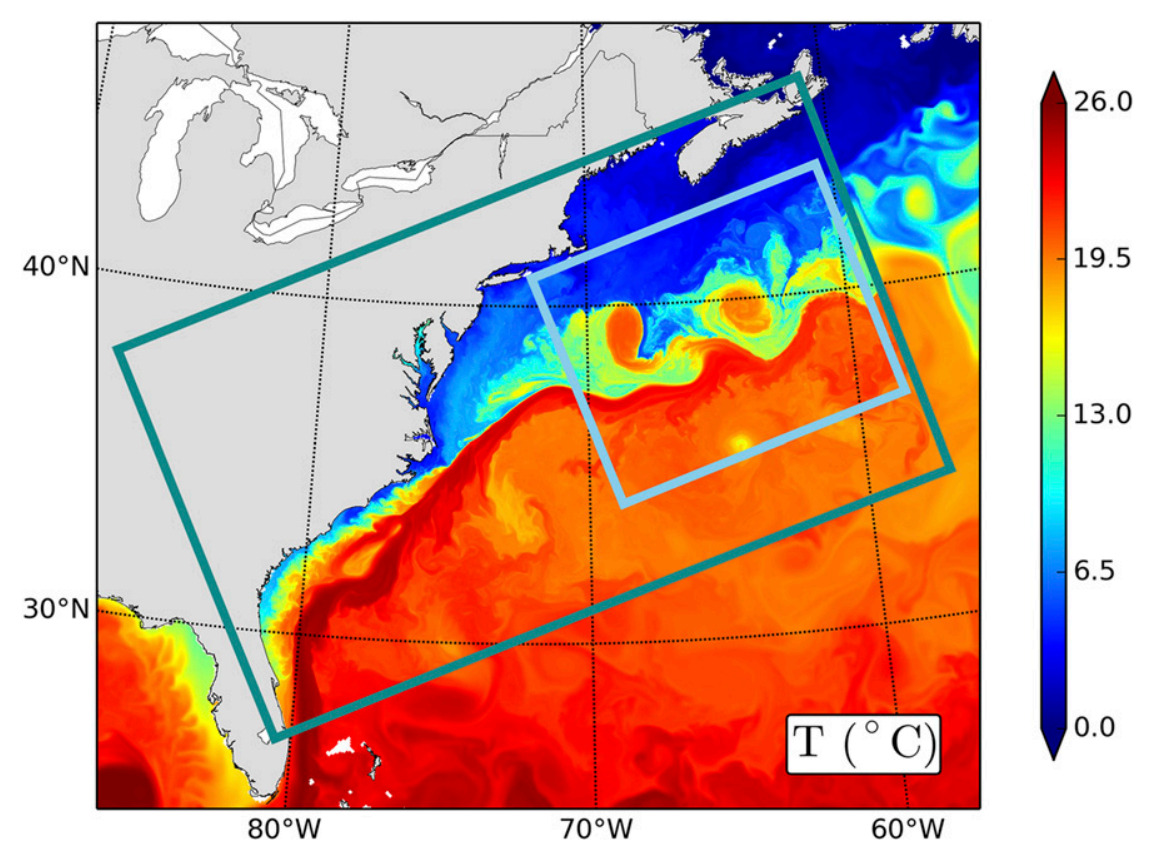

FIG. 3. Instantaneous SST in the region of the Gulf Stream at the end of winter as simulated by ROMS. The parent domain covers most of the Atlantic Ocean. The boundaries of the successively nested subdomains are delineated by thick colored lines. The SST plotted inside each of the domains is computed using data at the corresponding resolution. 

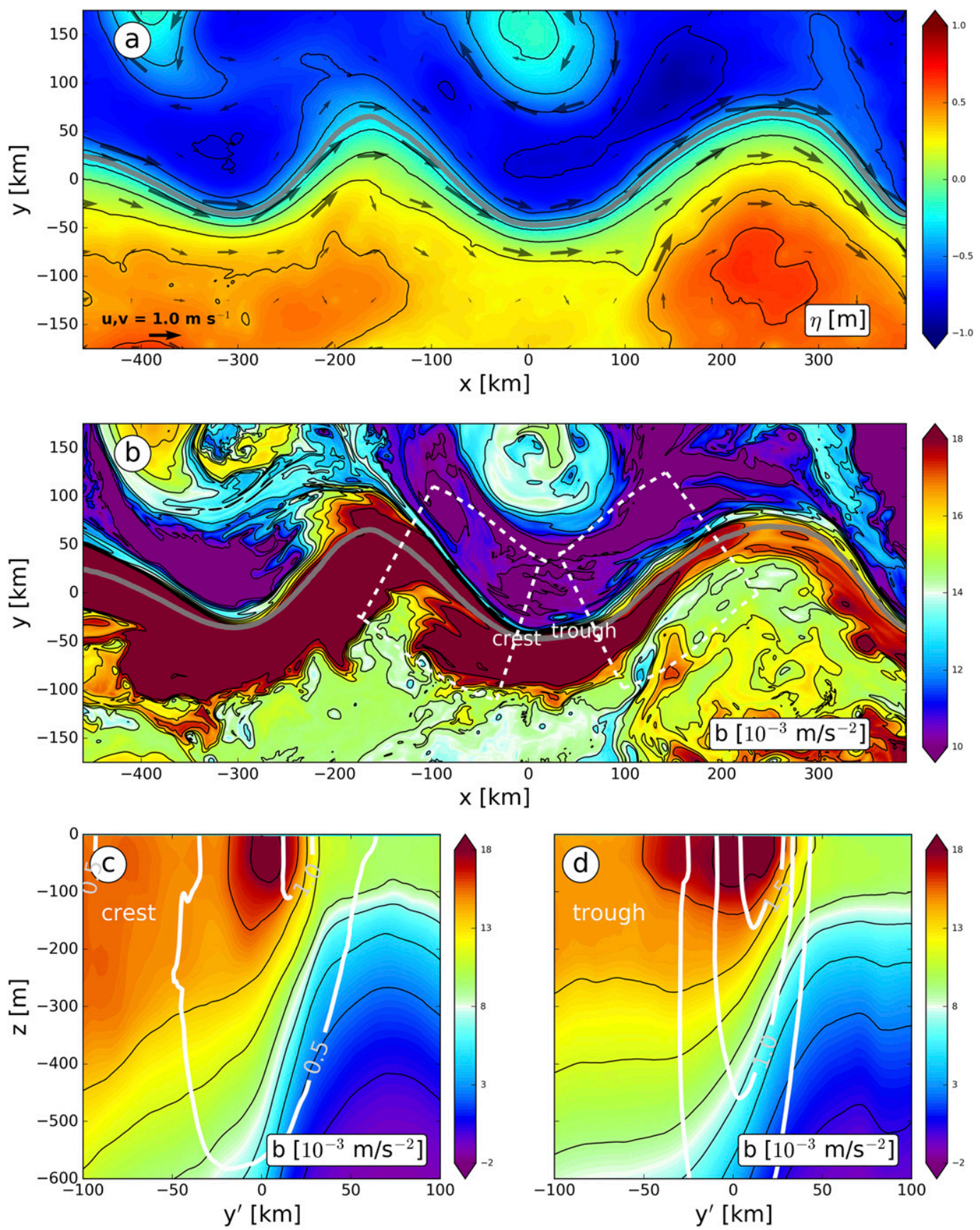

FIG. 4. Snapshot of the simulated meandering Gulf Stream downstream from separation. (a) SSH $\eta(\mathrm{m})$, (b) surface buoyancy $b\left(\mathrm{~m} \mathrm{~s}^{-2}\right)$, and vertical sections of buoyancy (colors) and along-stream velocity ( $\mathrm{m} \mathrm{s}^{-1}$; white contours) averaged in the along-stream direction over two regions representative of (c) a crest and (d) a trough, as defined by the dashed white contours in (b). The solid gray line in (a) and (b) is the approximate jet center at the surface defined by $\eta=0.3 \mathrm{~m}$.

positioned near the steepest pycnocline gradients, and larger amplitudes for larger meander meridional excursions. Areas of large $w$ typically have similar crossand along-stream scales of about $100 \mathrm{~km}$. Thomas and Joyce (2010) observe subduction occurring primarily in the uptrough sector, where the cross-stream velocity is confluent and frontogenetic [e.g., as in the Hoskins and Bretherton (1972) theory]; using a variant of the omega equation, they estimate a vertical velocity driven by the confluent flow in the vicinity of the intrusion 

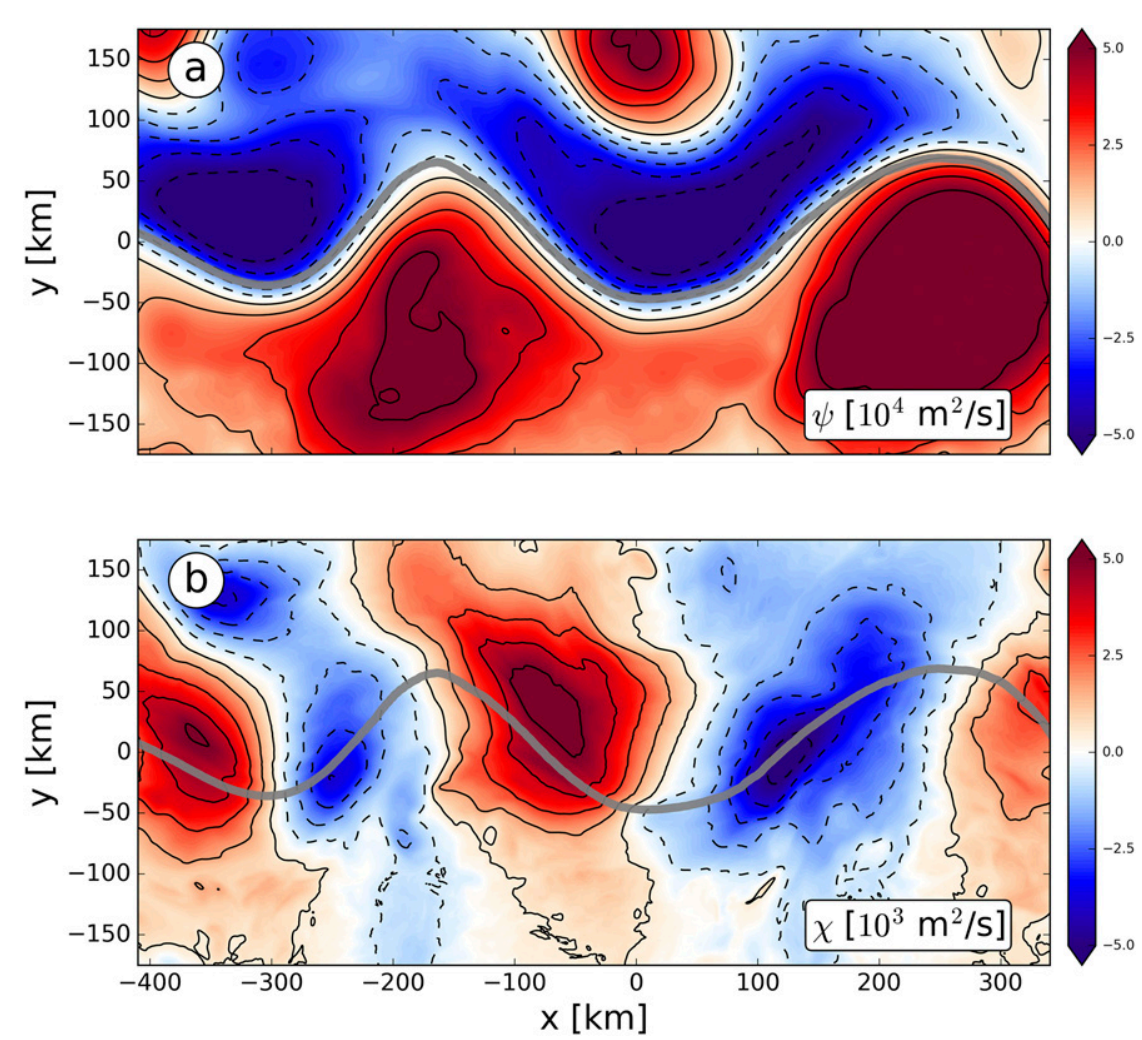

FIG. 5. (a) Rotational streamfunction $\psi$ and (b) divergent velocity potential $\chi$ at the surface for the simulated meandering Gulf Stream of Fig. 4 . The solid gray lines are the approximate jet center at the surface defined by $\eta=0.3 \mathrm{~m}$.

spanning 20-45 $\mathrm{m} \mathrm{day}^{-1}\left(3-5 \times 10^{-4} \mathrm{~m} \mathrm{~s}^{-1}\right)$. Downtrough, the flow is diffluent, driving an inferred vertical circulation in the opposite sense to that along uptrough sector.

The horizontal velocity can be evaluated in an incompressible fluid using a Helmholtz decomposition with two scalar potentials: $\psi$ is the rotational streamfunction and $\chi$ the divergent potential, as defined below in (2); relative to velocity or velocity gradient fields, these potentials accentuate the larger (mesoscale) features of the flow. Variable $\chi$ is an order of magnitude smaller than $\psi$ (Fig. 5); this is to be expected for a flow in approximate geostrophic balance, and this ordering is the theoretical basis for making a force-balanced $\mathrm{BE}$ model (section 4). The patterns of $\chi$ are tightly linked to the phase of the meanders, with extrema on the meander faces. There is convergence uptrough and divergence downtrough. Vertical velocities show the same relation with the phase of the meanders (Fig. 6). The surface convergence in the uptrough sector implies a downwelling $w$ underneath that reaches deep into the pycnocline (Fig. 6b); in the downtrough sector, these signs are reversed (Fig. 6c).

At greater depth in the ROMS simulation (not shown), the same meander patterns are evident but much weaker: at $z=-2000 \mathrm{~m}$, the peak value for $\psi$ is about $10^{3} \mathrm{~m}^{2} \mathrm{~s}^{-1}$, and for $\chi$ it is about $10^{2} \mathrm{~m}^{2} \mathrm{~s}^{-1}$; both magnitudes are about 50 times smaller than in Fig. 5. This is qualitatively consistent with the simulation results in Schubert et al. (2018) and with its included observational references. Furthermore, the deep baroclinicity is even weaker in the simulated meander pattern, with $|\nabla b|$ negligible compared to the upper ocean and no evidence of a deep frontal structure across the Gulf Stream.

In addition to showing the meander-scale structure, the instantaneous patterns of relative vorticity $\zeta / f$ and divergence $\delta / f$ highlight the strong submesoscale frontal dynamics in the region (Figs. 7a,b). The sharp North Wall front is instantaneously composed of multiple submesoscale fronts that appear as elongated vorticity filaments. However, the horizontal gradients of buoyancy and horizontal velocity also seem to be modulated by the phase of the meanders. The fronts and filaments are sharper in the uptrough sectors, and weaker in the downtrough sectors, as observed in the satellite images (Fig. 1). This modulation is characteristic of a meander-driven frontogenetic tendency in the uptrough sectors and a frontolytic tendency in the downtrough sectors. 

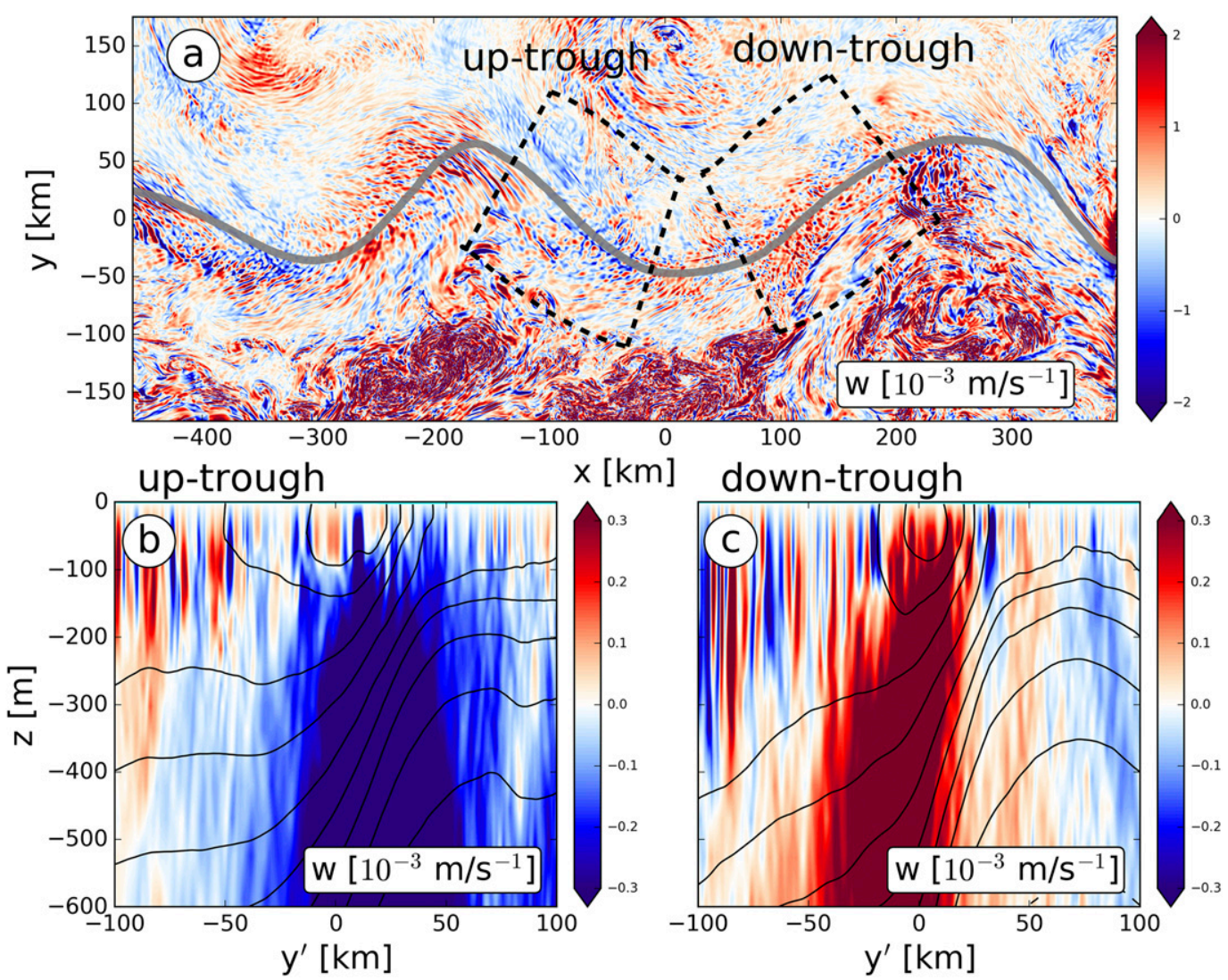

FIG. 6. (a) Vertical velocity at $z=-100 \mathrm{~m}$ depth for the simulated meandering Gulf Stream of Fig. 4. Vertical sections of vertical velocity (colors) and buoyancy (black contours) averaged in the along-stream direction over two regions representative of (b) an uptrough sector and (c) a downtrough sector as defined by the dashed black boxes in (a). The solid gray line in (a) is the approximate jet center at the surface defined by $\eta=0.3 \mathrm{~m}$.

\section{Balance equations diagnostic model}

The starting point for developing the SCFT dynamical theory is the conservative, hydrostatic PEs with a Boussinesq approximation for mass conservation and pressure gradient force and a $\beta$-plane approximation for the Coriolis frequency, $f=f_{0}+\beta y$. We decompose the buoyancy field into a horizontally and temporally averaged part and its deviation,

$$
b(x, y, z, t)=\bar{b}(z)+b^{\prime}(x, y, z, t) .
$$

Parameters $(x, y)$ are (east, north) horizontal coordinates, $z$ is upward (antiparallel to gravity), and $t$ is time. Parameter $b$ is in hydrostatic balance with the pressure, and the sea surface height anomaly $\eta$ is determined by a hydrostatic integration to the surface after subtracting its horizontal mean value. The purpose of the decomposition is to clearly distinguish a background "resting" stratification in $\bar{b}$ from its dynamical component $b^{\prime}$.

Asymptotically in the small Rossby number (Ro), the leading-order approximation is quasigeostrophy (QG), and it is "force balanced" in that it assumes the acceleration is small compared to other forces; in particular, it excludes inertia-gravity waves. Because Ro is not small in the Gulf Stream (section 3), QG is not reliably accurate, as is further demonstrated in section 6. A secondorder, energy-conserving, force-balanced model is the so-called balance equations, first identified by Lorenz (1960) and formally analyzed in McWilliams and Gent (1980). It has been shown to often be an accurate approximation for atmospheric and oceanic, rotating, stratified flows with moderate Ro values (most recently in the reduced-order study of fast oscillations around a "slow manifold" evolution; Chekroun et al. 2017). Its accuracy is comparable to other well-conceived, secondorder (in Ro), force-balanced models (cf. McWilliams et al. 2003).

\section{a. BE-SCFT equations}

The essential approximation in the BE, relative to the $\mathrm{PE}$, is the assumption that in a Helmholtz decomposition of the horizontal velocity, 

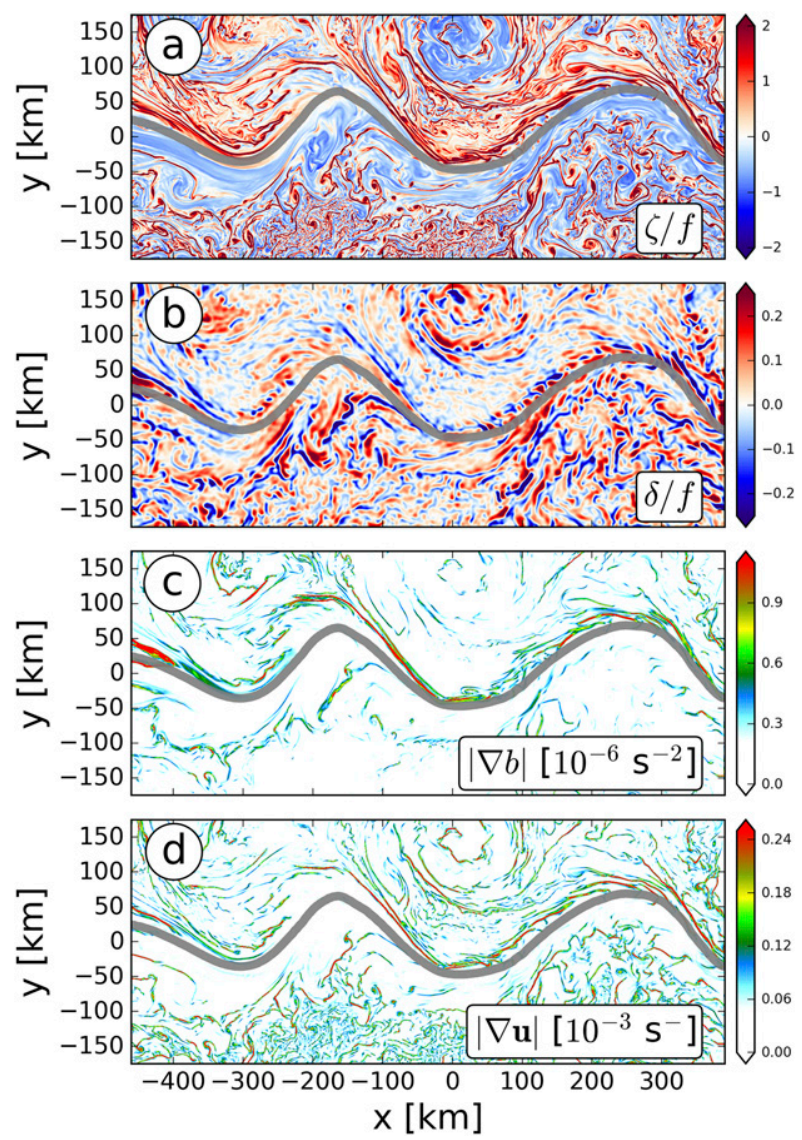

FIG. 7. Surface fields: (a) normalized vertical relative vorticity $\zeta / f$, (b) normalized horizontal divergence $\delta / f$ (smoothed using a convolution with a $20-\mathrm{km}$ half-width Gaussian kernel to emphasize the mesoscale pattern in the presence of internal waves and submesoscale currents), (c) horizontal gradient of buoyancy $|\nabla b|\left(\mathrm{s}^{-2}\right)$, and (d) horizontal gradient of horizontal velocity $|\nabla \mathbf{u}|\left(\mathrm{s}^{-2}\right)$ at the surface for the simulated meandering Gulf Stream of Fig. 4. The solid gray line is the approximate jet center at the surface defined by $\eta=0.3 \mathrm{~m}$.

$$
\mathbf{u}=\hat{\mathbf{z}}_{3} \times \nabla \psi+\nabla \chi,
$$

the rotational streamfunction $\psi$ is larger than the divergent potential $\chi$, specifically referring to their respective time derivatives in the vertical curl and horizontal divergence of the momentum equations. ${ }^{2}$ Vectors are in boldface

\footnotetext{
${ }^{2}$ The BE model choice made here differs from the diagnostic forcebalanced approximations in McWilliams (2017) and McWilliams (2018). There the essential assumption is that ageostrophic acceleration is neglected, rather than the neglect of divergent acceleration in the BE. This is because submesoscale currents approximately satisfy a turbulent thermal wind horizontal momentum balance between vertical momentum mixing, baroclinic pressure gradient, and Coriolis force, which has the effect of making $\psi$ and $\chi$ more similar in magnitude than typically occurs in a meandering pycnocline jet beneath the surface boundary layer.
}

and are purely horizontal unless indicated by a subscript 3 , and $\hat{\mathbf{z}}_{3}$ is the vertical unit vector. In an asymptotic nondimensionalization, $\chi$ is smaller than $\psi$ by $\mathscr{O}(\mathrm{Ro})$.

There are alternative equivalent ways to express the BE system. First define companion fields, the vertical vorticity $\zeta$, horizontal divergence $\delta$, and vertical velocity $w$, from which $\psi$ and $\chi$ are obtained by solving the horizontal elliptic equations,

$$
\nabla^{2} \psi=\zeta \text { and } \nabla^{2} \chi=\delta=-\partial_{z} w,
$$

with suitable lateral boundary conditions (described at the end of this section).

$\mathrm{BE}$ time-stepping is accomplished by solving a coupled system with three dependent fields, $\zeta, \partial_{t} \zeta$, and $w$, with a separate governing equation for each of them.

The equation for $\zeta$ is the divergence of horizontal momentum after neglecting terms involving $\chi$ or $w$ (consistent with second-order accuracy), denoted by DIV:

$$
\begin{aligned}
f_{0} \zeta & =\nabla^{2} \int_{-H}^{z} b^{\prime} d z+\mathscr{R}^{\mathrm{DIV}}, \\
\mathscr{R}^{\mathrm{DIV}} & =-\beta \nabla \cdot y \nabla \psi-2 J\left[\partial_{x} \psi, \partial_{y} \psi\right] .
\end{aligned}
$$

Parameter $J$ is the horizontal Jacobian operator. DIV is often referred to as gradient-wind balance when the nonlinear term in $\mathscr{R}^{\mathrm{DIV}}$ is large. The first right-side term is based on an assumption that the horizontal pressure gradient vanishes at the domain bottom at $z=-H$, with an accompanying vertical integration of hydrostatic balance. Another assumption here is that $\beta l / f_{0}$ is a small parameter of $\mathscr{O}(\mathrm{Ro})$ (a traditional assumption in $\mathrm{QG}$ theory), hence $\mathscr{R}^{\mathrm{DIV}}$ is of this order relative to the buoyancy term in (4). The QG approximation to DIV neglects $\mathscr{R}^{\mathrm{DIV}}$ and yields the geostrophic streamfunction, velocity, and vorticity,

$$
\psi_{g}=\frac{1}{f_{0}} \int_{-H}^{z} b^{\prime} d z, \quad \mathbf{u}_{g}=\hat{\mathbf{z}}_{3} \times \nabla \psi_{g}, \quad \zeta_{g}=\nabla^{2} \psi_{g} .
$$

In general the velocity is decomposed into geostrophic and ageostrophic components,

$$
\mathbf{u}=\mathbf{u}_{g}+\mathbf{u}_{a}, \quad \psi=\psi_{g}+\psi_{a}, \quad \nabla \cdot \mathbf{u}_{a}=\nabla^{2} \chi=-\partial_{z} w .
$$

Thus, $\chi$ is entirely ageostrophic, while $\psi$ has both geostrophic and ageostrophic components.

The BE equation for $\partial_{t} \zeta$ is the curl of the horizontal momentum equation, dubbed VORT,

$$
\begin{aligned}
\partial_{t} \zeta & =-\beta \partial_{x} \psi+f_{0} \partial_{z} w-J[\psi, \zeta]+\mathscr{R}^{\mathrm{VORT}}, \\
\mathscr{R}^{\mathrm{VORT}} & =-\beta \nabla \cdot(y \nabla \chi)-\nabla \cdot\left(\zeta \nabla \chi+w \nabla \partial_{z} \psi\right) .
\end{aligned}
$$


Parameter $\mathscr{R}^{\text {VORT }}$ contains $\psi-\chi$ cross terms in its advection operator, but no $\chi-\chi$ terms, again consistent with second-order accuracy.

The BE equation for $w$ is a linear combination of $-f_{0} \partial_{z}$ VORT $+\partial_{t} \partial_{z}$ DIV $+\nabla^{2}$ (buoyancy equation), which yields the so-called Omega equation, $\Omega E$ :

$$
\begin{aligned}
{\left[f_{0}^{2} \partial_{z}^{2}+N^{2}(z) \nabla^{2}\right] w=} & f_{0} \beta \partial_{z} \partial_{x} \psi+f_{0} \partial_{z} J[\psi, \zeta] \\
& -\nabla^{2} J\left[\psi, b^{\prime}\right]+\mathscr{R}^{\Omega E} \\
\mathscr{R}^{\Omega E}= & -f_{0} \partial_{z} \mathscr{R}^{\mathrm{VORT}}+\partial_{t} \partial_{z} \mathscr{R}^{\mathrm{DIV}} \\
& -\nabla^{2}\left(\nabla \chi \cdot b^{\prime}+w \partial_{z} b^{\prime}\right) .
\end{aligned}
$$

The mean stratification is $N^{2}(z)=\partial_{z} \bar{b}(z)>0$. No further approximations beyond those in DIV and VORT are made in deriving $\Omega E$.

The QG approximations to VORT and $\Omega E$ involve neglecting the $\mathscr{B}$ terms, replacing $\psi$ by $\psi_{g}$, and retaining the second right-side term in VORT using the geostrophic approximation to the $\Omega E$ solution, which is designated as $w_{\mathrm{ga}}$, with its accompanying $\chi_{\mathrm{ga}}$. These subscripts are a reminder that, although $\nabla \chi_{\mathrm{ga}}$ and $w_{\mathrm{ga}}$ are ageostrophic velocities, they are ones consistently calculated at leading order in Ro from $\psi_{g}$.

In the BE-SCFT diagnostic model the BE are not integrated forward in time; nevertheless the coupled system, (3)-(4) and (7)-(8), has to be solved at a given instant to determine $\psi$ and $\chi$, hence $\mathbf{u}, \zeta$, and $w$. This is done by successive iteration among them until convergence, starting with $\psi=\psi_{g}$ and $\chi=0$. Because DIV does not depend on $\chi$ or $\partial_{t} \psi$, it can be iterated to convergence in $\psi$ independent of a second-stage iteration between VORT and $\Omega E$ for $\partial_{t} \psi$ and $\chi$. The QG system breaks this coupling: $\psi_{g}$ is directly determined in (5), then $\Omega \mathrm{E}$ gives $w_{\mathrm{ag}}$, and finally $\partial_{t} \psi_{\mathrm{g}}$ can be evaluated from VORT.

As in all higher-order force-balanced models, there are solvability limits, here expressed as nonconvergence of the iterations, although the latter is not proof of the former. In this paper all BE-SCFT solutions are necessarily within the solvable regime, but for larger Ro values and some more extreme shape parameters (specified in section 5) than reported here, BE-SCFT solutions of the discretized system failed to converge. For the continuous BE system, three solvability failure conditions are identified through second-order in Ro: unstable stratification $\left(N^{2}+\partial_{z} b^{\prime}<0\right)$, violation of centrifugal stability $(f q<0$, where $q$ is Ertel potential vorticity), and a loss of solvability for DIV $\{f(A-S)<0$ where $A=f+\zeta$ is the absolute vorticity and $S$ is the horizontal strain rate magnitude associated with the rotational flow component, $\left.S=\left[\left(\partial_{x}^{2} \psi-\partial_{y}^{2} \psi\right)^{2}+4\left(\partial_{x} \partial_{y} \psi\right)^{2}\right]^{1 / 2}\right\}($ Yavneh et al. 1997; McWilliams et al. 1998). For small Ro (i.e., QG), none of these failure conditions will occur, and for our BE solutions they are assessed a posteriori in section $6 \mathrm{~d}(1)$. In a progression with Ro increasing from a small value, the third condition is usually the most vulnerable to being triggered. When the BE fail, unbalanced behaviors (e.g., internal waves) are expected, and for each failure condition unbalanced instability modes have been identified (McWilliams et al. 2003). Nevertheless, experience with realistic simulations (as in section 3 ) shows that the flow behavior often remains close to force-balanced dynamics even beyond its strict realm of validity. Calculating a consistent diagnostic BE-SCFT solution is less demanding with respect to its solvability than is making an extended BE time integration when Ro is not small.

\section{b. Frontogenetic tendency equations}

The preceding indicates how $\mathbf{u}_{g}$ and $\left(\mathbf{u}_{a}, w\right)$ are diagnostically determined from $(b, \eta)$ by making balanceddynamics approximations; this comprises the secondary circulation (SC) analysis. For the FT analysis, no approximations are needed relative to the PEs, so none are made. Frontogenesis is defined as a sharpening of the horizontal gradients of either buoyancy or horizontal velocity. The derivation of the FT relations is made by the operation of $\nabla A \cdot \nabla$ on the $D A / D t$ equation, where $A=u, v$, and $b^{\prime}$ in turn. Then the resulting equations for $A=u$ and $v$ are added together to obtain a total horizontal velocity gradient variance relation. The results are the FT equations:

$$
\begin{aligned}
& \frac{D}{D t}\left[\frac{1}{2}\left(\partial_{i} u^{j}\right)\left(\partial_{i} u^{j}\right)\right]=\mathscr{T}^{\mathbf{u}}=\beta\left(v \partial_{y} u-u \partial_{y} v\right)-\left(\partial_{i} u^{j}\right)\left(\partial_{i} \partial_{j} \phi\right)-\left(\partial_{i} u^{j}\right)\left(\partial_{i} u^{k}\right)\left(\partial_{k} u^{j}\right)-\left(\partial_{i} u^{j}\right)\left(\partial_{i} w\right)\left(\partial_{z} u^{j}\right), \\
& \frac{D}{D t}\left[\frac{1}{2}\left(\partial_{i} b^{\prime}\right)\left(\partial_{i} b^{\prime}\right)\right]=\mathscr{T}^{b}=-\left(\partial_{i} b^{\prime}\right)\left(\partial_{i} u^{j}\right)\left(\partial_{j} b^{\prime}\right)-\left(\partial_{i} b^{\prime}\right)\left(\partial_{i} w\right)\left(\partial_{z} b\right) .
\end{aligned}
$$

The compact notation used here is a sum over a repeated index, and the indices $(i, j, k)$ span $(x, y)$ (horizontal). The interpretation of FT is that the Lagrangian time derivative of the positive square of a horizontal-gradient vector changes according to the right-side terms, all of which involve velocity gradient factors. For $\mathscr{T}^{\mathbf{u}}$ the right-side influences are due, respectively, to $\beta$, the pressure-gradient force, and both horizontal and vertical 
advection. For $\mathscr{T}^{b}$ the only influence is advection. Positive terms in (9) indicate a frontogenetic tendency.

For a purely geostrophic flow, $\mathbf{u}=\mathbf{u}_{g}$ and $w=0$, $\mathscr{T}^{\mathbf{u}}=0$. Furthermore, for Ro $\sim 1$ and highly anisotropic flow patterns, such as a meandering Gulf Stream whose width is narrow compared to the meander wavelength, the frontogenetic rate associated with FT-defined as

$$
\frac{D}{D t} \log \left[\frac{1}{2}(\nabla A)^{2}\right]=\frac{\mathscr{T}^{A}}{\frac{1}{2}(\nabla A)^{2}}
$$

-is approximately equal to $-2 \delta$ to leading order in the horizontal anisotropy scale ratio for both $\mathscr{T}^{\mathbf{u}}$ and $\mathscr{T}^{b}$ (Barkan et al. 2018, manuscript submitted to J. Phys. Oceanogr.); that is, strong frontogenesis is controlled by the ageostrophic horizontal convergence rate, especially at the surface where the Gulf Stream front is sharpest (sections 5 and 6 ).

\section{c. Numerical methods}

The BE and FT equations are discretized with secondorder accuracy in space. The horizontal grid has uniform $d x=d y$, and the vertical grid is stretched in $z$ between $-H$ and 0 with smaller $d z$ near the surface.

The 2D elliptic equations in (3) and the 3D equation in $(8)$ are solved with cross-jet $(x)$ boundary conditions of zero normal derivative, along-jet $(y)$ boundary conditions of periodicity, and vertical boundary conditions of $w=0$ at $z=-H$ and 0 . The $x$ condition is thus one of vanishing far-field flow. The $z$ conditions are vanishing flow at depth and a rigid-lid approximation at the surface. MATLAB matrix inversion routines are used to solve these discretized elliptic equations. This imposes a constraint on the size of the 3D spatial grid, and thus limits the narrowness of the North Wall front that can be specified in the idealized $b$ shape in section 5 while obtaining accurate BE-SCFT solutions.

The BE iterations among the DIV, VORT, and $\Omega E$ subsystems are made with relaxation parameters to improve the convergence rate; that is, for a new estimate of a field $A^{n+1}$ obtained by evaluating its governing equation, it is replaced by a composite estimate, $\gamma A^{n+1}+(1-\gamma) A^{n}$, for a positive $\gamma \leq 1$ chosen experimentally.

\section{Idealized meandering jet shape}

The conservative SCFT program is to specify a shape for $b(x, y, z)$, then diagnose the $3 \mathrm{D}$ circulation $\mathbf{u}_{3}$ and frontogenetic tendencies $\mathscr{T}$ (section $4 \mathrm{~b}$ ). In this section the procedure for specifying $b$ is presented.

The Gulf Stream is a turbulent jet, and as such no realization is the same: you could not step twice in the same river (Heraclitus). Nevertheless, we can propose an idealized, typical shape, guided by sparse observations and short simulations with a limited number of realizations. From the literature on the jet stream and Gulf Stream (sections 1 and 2) and the oceanic simulation analysis (section 3), the following seem to be salient characteristics of an oceanic eastward jet:

- surface intensification of the velocity;

- meridional meandering $(m)$ of the jet center;

- narrower trough width $(w)$ than crest;

- cross-jet asymmetry $(a)$ with narrower polar side than equatorial side;

- equatorward tilt $(t)$ of the horizontal front with increasing depth; and

- a buoyant surface-layer jet core $(*)$, sometimes.

(The parenthetical symbols match parameter definitions below.) All of these characteristics are shared with the tropospheric jet stream with its peak velocity at the tropopause, except for the last one (Keyser and Shapiro 1986).

The particular characteristic of a narrower trough implies horizontally confluent flow along the uptrough sector and diffluent flow downtrough. From the classical perspective of strain-induced frontogenesis (Hoskins and Bretherton 1972), we can therefore expect frontogenesis and frontolysis, respectively, in these sectors. The other shape characteristics have not been assessed for their SCFT implications.

We now define functional forms to express these shape features. It is, of course, somewhat arbitrary which functional forms are chosen to represent the jet shape, but in our judgment the qualitative BE-SCFT behaviors obtained in section 4 are not strongly sensitive to the functional choices.

A further consideration is that this idealized meandering jet also should be potentially unstable to mesoscale perturbations, as the Gulf Stream region is well known to be a source of eddy generation. Because the assumed jet shape has a central maximum and strong shear, both vertically and horizontally, we can be confident that it satisfies the necessary conditions for QG baroclinic and/or barotropic instability (i.e., sign changes in the horizontal gradient of potential vorticity). The resulting eddying behavior is apparent in the ROMS simulations (section 3) whose meander shape is being mimicked here; however, an instantaneous, diagnostic SCFT analysis does not yield the possible unstable modes as part of its solution, which therefore is a separate aspect of the general flow evolution.

The BE-SCFT analysis domain is $\left[-(1 / 2) L_{x},(1 / 2) L_{x}\right]$ in $x$ (the periodicity direction), $\left[-(1 / 2) L_{y},(1 / 2) L_{y}\right]$ in $y$ (the cross-jet direction), and $[-H, 0]$ in $z$. For the domain size and the Coriolis frequency we choose 


$$
\begin{aligned}
L_{x} & =400 \mathrm{~km}, \quad L_{y}=350 \mathrm{~km}, \quad f_{0}=0.781 \times 10^{-4} \mathrm{~s}^{-1}, \\
\beta & =1.83 \times 10^{-11} \mathrm{~m}^{-1} \mathrm{~s}^{-1} .
\end{aligned}
$$

The latter corresponds to a latitude of $34^{\circ} \mathrm{N}$, which in retrospect is erroneously somewhat small, though inconsequential for our analyses and conclusions here.

We choose a jet shape with flow only in the upper ocean $(z>-H)$ with a characteristic depth scale comparable to the pycnocline depth $h_{p}(\ll H)$ and with a weak stratification anomaly in the surface layer whose depth scale is $h_{s}<h_{p}$. This neglects the abyssal expression of the meandering Gulf Stream, which is described for the ROMS simulations in section 3b. Alternatively expressed, there is a neglected, additive "reference level" horizontal velocity at $z=-H$ that would tend to enhance the upper ocean flow pattern, but it is assumed to be mostly geostrophic, have little baroclinicity, and contribute nothing significant to the upper ocean SCFT behaviors that are our main focus in this paper.

The buoyancy shape is defined in relation to two independent stratification profiles on the southern and northern sides of the jet, $\bar{b}_{S}(z)$ and $\bar{b}_{N}(z)$, taken from the ROMS simulation in the meandering sector (section 3 ). These are used to define the mean buoyancy and meridional buoyancy difference profiles:

$$
\bar{b}(z)=\frac{1}{2}\left(b_{S}+b_{N}\right), \quad \Delta \bar{b}(z)=b_{N}-b_{S} .
$$

The simulation $b$ profiles are fit with analytic functions be smooth and differentiable, as well as to be consistent with zero meridional gradient at the bottom of the domain $(z=-H)$; that is, $\bar{b}_{S}(-H)=\bar{b}_{N}(-H)$, hence $\Delta \bar{b}(-H)=0$ (appendix A). The buoyancy profiles are plotted in Fig. 8. Both $b_{S}$ and $b_{N}$, hence also $\bar{b}$, are monotonically increasing with height, consistent with stable stratification and surface intensification of the geostrophic velocity $\mathbf{u}_{g}$; furthermore, $\Delta \bar{b}$ and $\mathbf{u}_{g}$ are positive everywhere, implying an entirely eastward flow and vertical shear.

The total buoyancy field is defined by

$$
\begin{aligned}
b(x, y, z)= & b_{S}(z)+\frac{1}{2} \Delta \bar{b}(z)\left\{\tanh \left[\frac{y_{c}(x, z)-y}{l(x, y, z)}\right]-1\right\} \\
& +b_{*} \exp \left[-\frac{z^{2}}{h_{*}^{2}}\right] \operatorname{sech}\left[\frac{y-y_{c}(x, z)}{l(x, y, z)}\right] .
\end{aligned}
$$

The first term is the southern reference profile; the second term is the primary jet shape with its $\partial_{y} b \leq 0$ (and its associated $u_{g} \geq 0$ ); and the third term is the buoyant jet core in the surface layer. The meandering jet center is at $y_{c}(x, z)$, and the meridional jet width

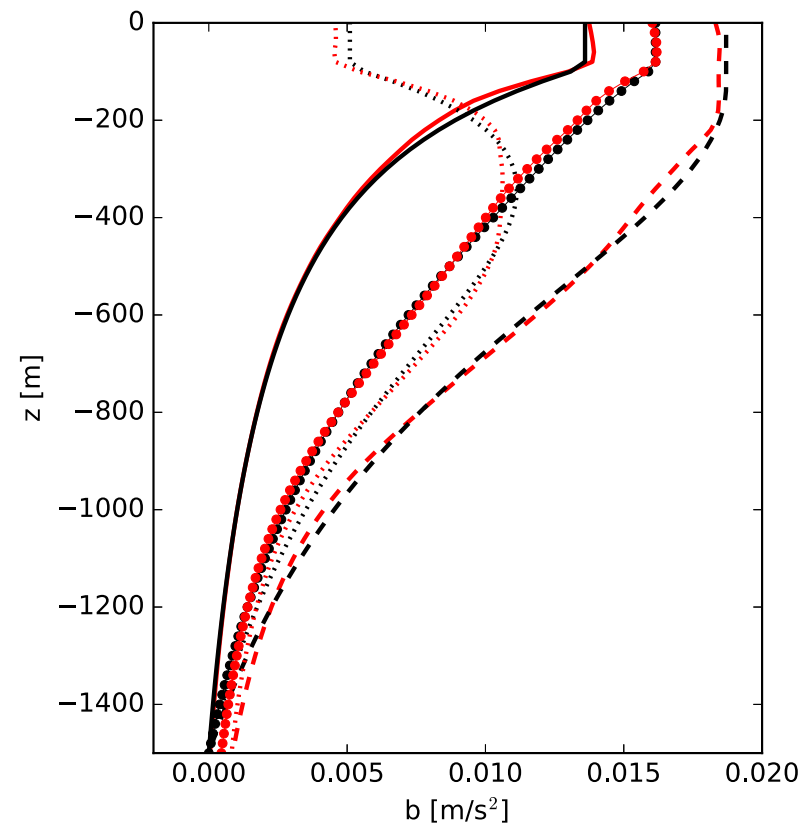

FIG. 8. Buoyancy profiles for an analytic fit using (A1) and (A2): $\bar{b}_{S}(z)$ (black dashed line) and $\bar{b}_{N}(z)$ (black solid), along with their average $\bar{b}(z)$ (black large dotted) and difference $\Delta \bar{b}(z)$ (black small dotted). Also plotted are their counterparts in red based on area averages for the ROMS solution in regions on either side of the separated Gulf Stream.

scale is $l(x, y, z)>0$. The mean jet position at the surface is at $y=0$. The analytic formulas for $y_{c}$ and $l$ are in appendix $\mathrm{B}$, along with dimensional parameters for mean jet width $l_{0}$ and the buoyant core $\left(b_{*}, h_{*}\right)$ and nondimensional parameters for meander amplitude $c_{m}$, width asymmetry along the jet $c_{a}$, width asymmetry across the jet $c_{w}$, and vertical tilt of the jet center $c_{t}$.

To analyze the upper-ocean flow in an idealized jet, the level $z=-H$ is assumed to be one with no motion, so by hydrostatic integration,

$\phi(x, y, z)=\phi_{0}+\int_{-H}^{z} b\left(x, y, z^{\prime}\right) d z^{\prime}, \quad \eta(x, y)=\frac{1}{g} \phi^{\prime}(x, y, 0)$,

with $\phi_{0}$ a constant and the surface elevation linearized about the mean sea level at $z=0$. The mean dynamic pressure profile $\bar{\phi}(z)$ is the hydrostatic integral in $z$ of $\bar{b}(z)$. This idealization thus neglects the abyssal flow in the Gulf Stream, presuming it has only a weak expression in the upper ocean; this is partly because the deep flow is probably not highly correlated with surface meanders.

The resulting $b$ field is shown in Fig. 9. At the surface the meander pattern is clearly seen along with the 

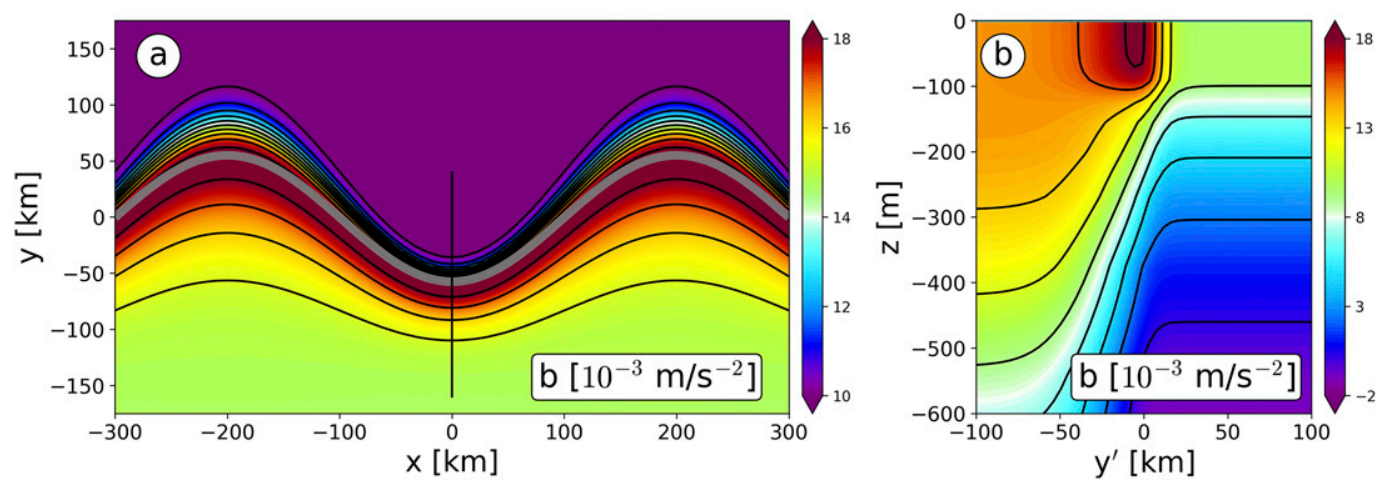

FIG. 9. Buoyancy field for the idealized shape in (13) and (B6): (a) surface $b(x, y, 0)$ and (b) cross-section $b\left(0, y^{\prime}, z\right)$ at the trough. The plotted $x$ domain is $\left[-(3 / 4) L_{x},(3 / 4) L_{x}\right]$ with periodic extension beyond the BE-SCFT domain width $L_{x}$. In (a) the gray line is the jet center curve at the surface $y_{c}(x, 0)$, and in (b) $y^{\prime}=y-y_{c}(0,0)$ is a northward coordinate relative to the jet center at the surface in the trough. Note the different amplitude ranges in the two plots.

narrower trough than crest and the narrower north side (the Wall) than south side. In a cross section at the trough, the equatorward tilt with depth is also evident. In the dynamic height gradient, $\nabla \phi^{\prime}$ and $g \nabla \eta$, and geostrophic velocity $\mathbf{u}_{g}$ (not shown), the jet center at the surface is displaced south of $y_{c}(x, 0)$ because of the tilt, $c_{t} \neq 0$; that is, the strongest subsurface $\nabla b$ values are on the equatorward side, and the hydrostatic integration (14) accumulates this effect.

While there is obviously a fair amount of complexity in these functional shapes and free parameters, the approach is to fit them approximately to the meandering jet structure in ROMS (section 3), and then systematically explore the sensitivities of the BE-SCFT solutions to the various shape attributes enumerated at the beginning of this section. The purpose is to understand why the meandering Gulf Stream has the SCFT behavior it does.

\section{Balanced diagnostic solutions}

The simplest jet configuration is a straight zonal flow $\left(c_{m}=0\right)$. From the perspective of this paper, this is a null case. The flow is geostrophic only, the conservative QG and BE solutions are the same, and there is no frontogenetic tendency. The other shape parameters $\left(c_{w}, c_{a}, c_{t}\right.$, and $\left.b_{*}\right)$ influence $b(x, z)$, hence also $\mathbf{u}_{g}(x, z)$, but they do not cause any SCFT effects.

A simplest meandering jet configuration is defined with $c_{m} \neq 0$ but $c_{w}=c_{a}=c_{t}=0$, either with or without the buoyant core $\propto b_{*}$. This does have a nontrivial SCFT outcome, and the QG and BE solutions differ. However, because this is not an accurate fit to the ROMS $b$ field in section 3, we do not expect its SCFT outcome to be accurate either, and its analysis is deferred until section $6 \mathrm{~b}$ after first examining the $b$ configuration specified in section 5 .

\section{a. A standard jet shape}

With the standard shape parameters in (13) and (B6), the diagnosed circulation fields are shown in Figs. 10-15, in each case by first showing the full BE field and then showing the BE-QG ageostrophic component. Measured by the peak vorticity at the surface (Fig. 11), the Rossby number $\mathrm{Ro}_{\zeta}=\zeta / f \approx 1.3$ is not small, so QG solutions are not expected to be accurate. In fact, the finite-Ro corrections are typically on the order of $25 \%$ of the BE solution amplitude (except for $w$ in the upper ocean, where the finite-Ro ageostrophic correction is nearly as large as the total), and the patterns are roughly anticorrelated; that is, the finite-Ro corrections tend to reduce the $\mathrm{BE}$ amplitudes compared to the QG ones, although this is not true at all points. Thus, many of the $\mathrm{BE}$ solution patterns are qualitatively similar in structure to the QG ones but with moderate reductions in amplitude.

The rotational flow at the surface ( $\psi$ and $\zeta$ in Figs. 10, 11) clearly shows the meander pattern and the relative narrowness of the jet in the trough and along the North Wall. Because of these asymmetries the cyclonic vorticity north of the jet center is much stronger than the anticyclonic vorticity to the south, and its peak occurs in the trough where there is cyclonic curvature in the meander pattern. Gradient-wind balance (4) for curved flows has a weaker $\psi$ minimum north of the trough and a stronger $\psi$ minimum north of the crest, relative to geostrophic balance, as seen in the extrema in $\psi_{a}$. This is because of the reinforcing signs of geostrophic and cyclostrophic forces around a cyclonic center (i.e., the trough), which acts to weaken the streamfunction 

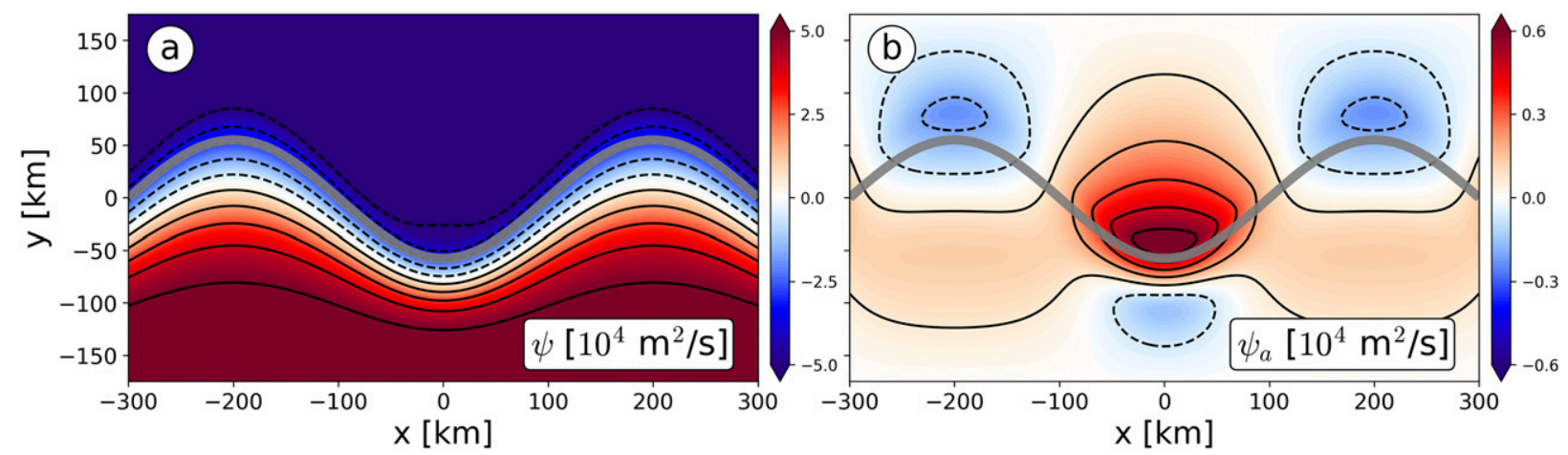

FIG. 10. Idealized streamfunction field $\psi$ at the surface determined from the BE SC analysis of $b$ in Fig. 9 for the standard shape parameters in (13) and (B6): (a) total $\psi(x, y, 0)$ and (b) ageostrophic $\psi_{a}(x, y, 0)$. The light gray line is the jet center at the surface $y_{c}(x, 0)$. Note the different amplitude scales in the two plots.

anomaly relative to the pressure anomaly. These two forces have opposing signs around an anticyclonic center (i.e., the crest), which strengthens the streamfunction relative to the pressure. The maximum $\psi_{a}$ north of the trough is the dominant finite-Ro ageostrophic correction to $\psi$, consistent with the combined asymmetries that occur there. Just as $\psi_{a}$ reduced $\psi$, so does $\zeta_{a}$ reduce $\zeta$. The patterns in $\psi$ and $\zeta$ are zonally symmetric about the meander trough and crest, as they must be because of the same symmetry in $b$.

A meridional section of $\zeta$ through the trough (where it is largest at the surface) shows a generally surfaceintensified shape, with stronger cyclonic vorticity to the north and weaker anticyclonic vorticity to the south. The equatorward and downward tilt of the jet is evident especially within the North Wall. The finite-Ro ageostrophic correction $\zeta_{a}$ is opposite to the total $\zeta$ because of gradient-wind balance.

The divergent velocity potential $\chi$ has its extrema on the meander faces, rather than at the meander trough and crest as $\psi$ has (Fig. 13). The uptrough face has a positive $\chi$ center somewhat closer to the trough than crest. This implies a surface convergence center with a downwelling $w$ underneath that reaches deep into the pycnocline (Figs. 14, 15); the most negative $w$ value occurs at $z=-525-\mathrm{m}$ depth. In the downtrough sector, these signs are reversed. There is zonal antisymmetry about the trough at $x=0$ in $\chi$ and $w$, again as expected from the even symmetry in $b$. The finite-Ro ageostrophic corrections, $\chi_{a}$ and $w_{a}$, are again mainly of opposite sign to the total ageostrophic fields, but now with substantial pattern differences. This is most evident in the uptrough cross section (Fig. 15), where $w_{a}$ weakens the downwelling in the North Wall and strengthens it and spreads it further south in the south side. Deeper in the pycnocline, $w_{a}$ is small relative to $w$, about $25 \%$. Overall, however, $\chi$ and $w$ have a reasonably deep vertical structure, hence $\delta$ at the surface (not shown) closely resembles $w$ in its pattern, as expected from continuity. The strongest $w$ values occur as a pair on either side of the trough. The magnitude of $\chi$ is small compared to $\psi$, in spite of the large value of $\mathrm{Ro}_{\zeta}$, which supports the
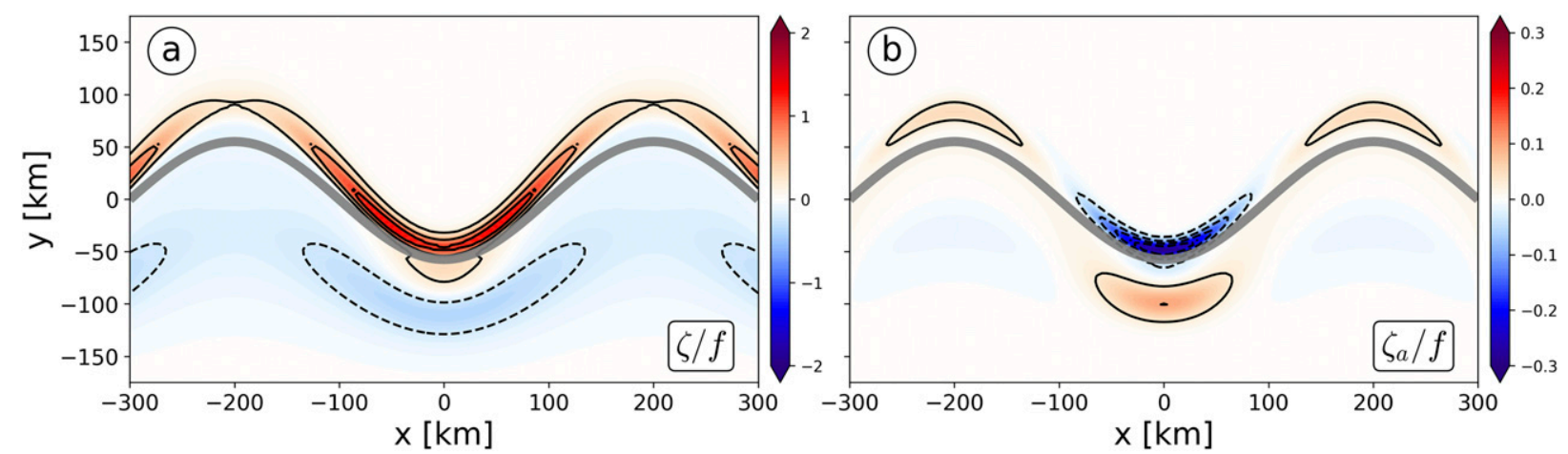

FIG. 11. Idealized normalized vertical vorticity field $\zeta / f_{0}$ at the surface determined from the BE SC analysis of $b$ in Fig. 9 for the standard shape parameters in (13) and (B6): (a) total $\zeta(x, y, 0) / f_{0}$ and (b) ageostrophic $\zeta_{a}(x, y, 0) / f_{0}$. The light gray line is the jet center at the surface $y_{c}(x, 0)$. Note the different amplitude scales in the two plots. 

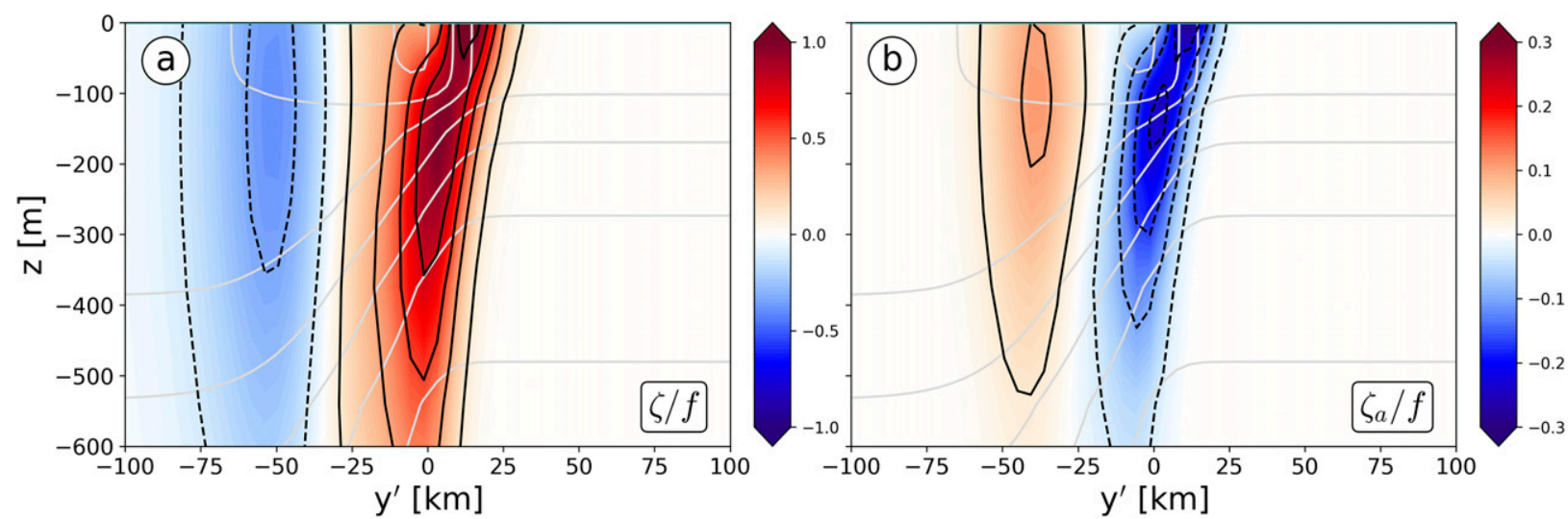

FIG. 12. Idealized normalized vertical vorticity field $\zeta / f_{0}$ in a cross section at the trough at $x=0$ determined from the BE SC analysis of $b$ in Fig. 9 for the standard shape parameters in (13) and (B6): (a) total $\zeta\left(0, y^{\prime}, z\right) / f_{0}$ and (b) ageostrophic $\zeta_{a}\left(0, y^{\prime}, z\right) / f_{0}$. The coordinate $y^{\prime}=y=y_{c}(0,0)$ is northward relative to the jet center at the surface in this section. Note the different amplitude scales in the two plots.

validity of the approximation justifying the $\mathrm{BE}$ model (section 4).

The pattern of $w$ has a simple, partial interpretation as zonal propagation in the sense that downwelling in the uptrough sector implies that the pycnocline is being depressed as the eastward-moving meander pattern brings warmer water past a point, and vice versa in the downtrough sector. If the solution had the functional form of

$$
e=E(x-c t, y, z)
$$

for all fields $e$, it would satisfy a wave equation,

$$
\partial_{t} e+c \partial_{x} e=0 .
$$

A diagnostic test of this behavior is in Fig. 16, which is a scatter plot of $\partial_{t} \psi$ versus $-\partial_{x} \psi$ for all $(x, y, z)$ points in the BE-SCFT solution. Perfect propagation would appear as a straight line whose slope is the zonal propagation speed $c$. This is evidently approximately true in some bulk sense with $c$ of about $0.22 \mathrm{~m} \mathrm{~s}^{-1}$, but obviously it is far from being an exact relation, implying there is much more to the solution evolution than simple propagation. We interpret this $c$ value as an eastward propagation tendency due to advection by the eastward jet. For comparison, if the meander behaved as a baroclinic Rossby wave in an otherwise resting fluid, $c$ would be negative (westward) and much smaller. This qualitative picture of downwelling on the north side of the uptrough sector and upwelling on the south side of the downtrough sector is broadly consistent with the simple kinematic model of Bower (1991).

The associated frontogenetic tendencies (9) are shown in Figs. 17 and 18. They clearly show frontogenesis in the uptrough sector along the North Wall in both buoyancy and velocity, with frontolysis along the downtrough sector that extends downward and equatorward along the tilted $b$ front. The velocity pattern $\mathscr{T}^{\mathbf{u}}$

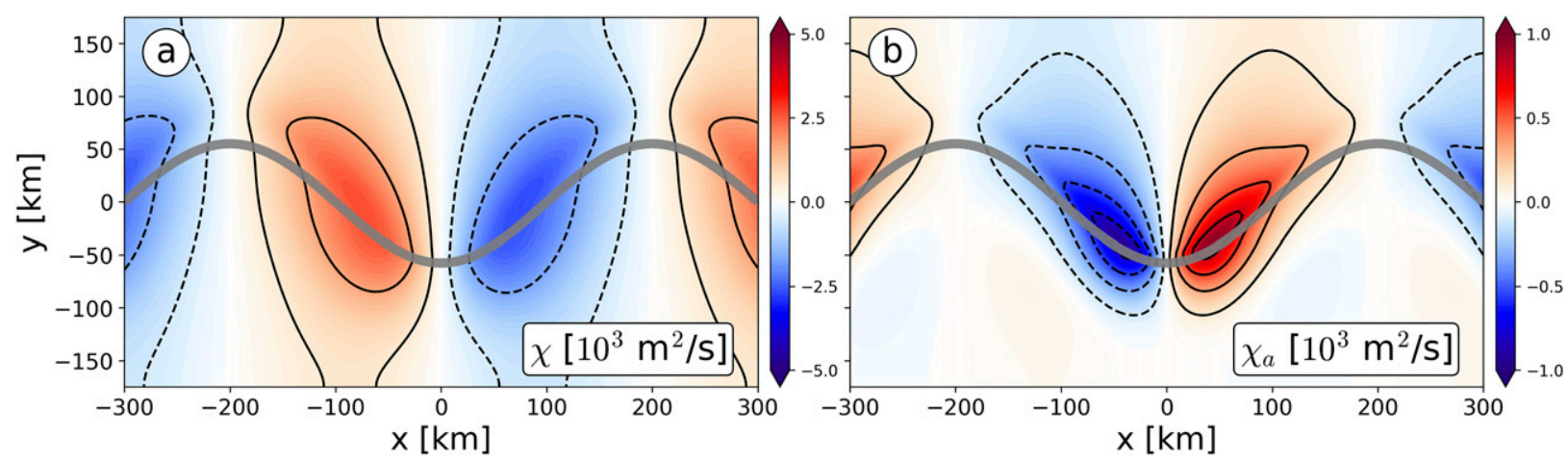

FIG. 13. Idealized divergent velocity potential $\chi$ at the surface determined from the BE SC analysis of $b$ in Fig. 9 for the standard shape parameters in (13) and (B6): (a) total $\chi(x, y, 0)$ and (b) ageostrophic $\chi_{a}(x, y, 0)$. The light gray line is the jet center at the surface $y_{c}(x, 0)$. Note the different amplitude scales in the two plots. 


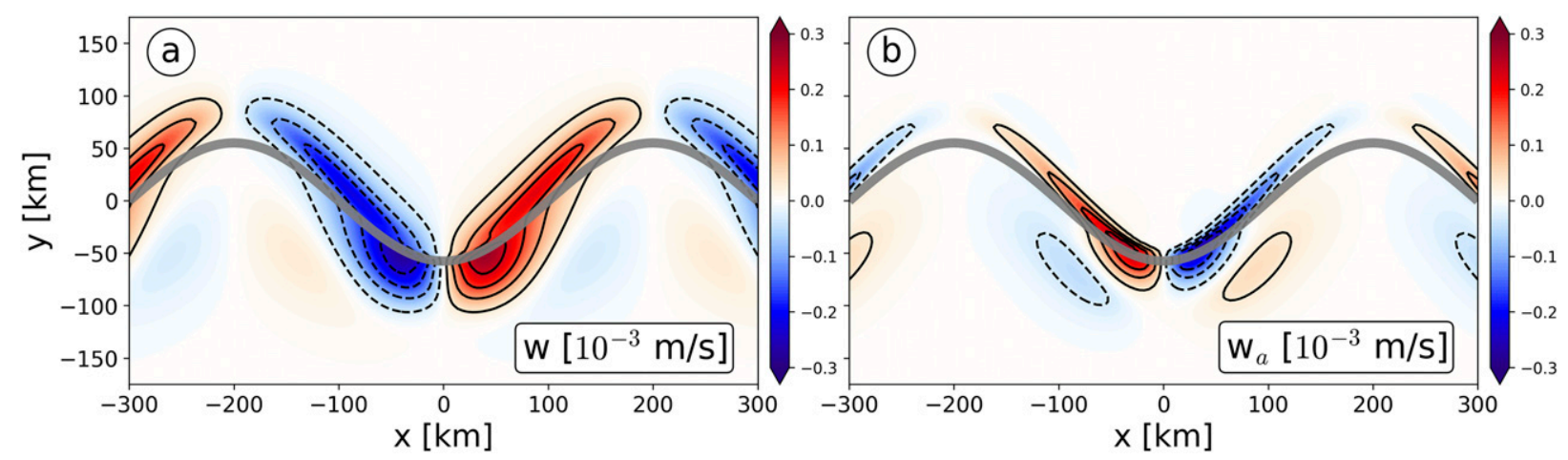

FIG. 14. Vertical velocity $w$ in the upper ocean at $z=-102 \mathrm{~m}$ determined from the BE SC analysis of $b$ in Fig. 9 for the standard shape parameters in (13) and (B6): (a) total $w(x, y,-114)$ and (b) ageostrophic $w_{a}(0, y,-114)$. The light gray line is the jet center at the surface $y_{c}(x, 0)$.

is particularly simple, while the buoyancy pattern $\mathscr{T}^{b}$ exhibits some complexity very near the trough. We would also expect some degree of balanced adjustment in the further evolution of the meandering jet, so that the $b$ and $\mathbf{u}$ fields will adjust toward mutual consistency between their respective frontogenetic tendencies.

This BE-SCFT analysis thus provides an explanation for the ageostrophic secondary circulation, as well as for the sharp North Wall in the uptrough sector of a meandering jet, the tilted jet front in $(y, z)$, and, after the fluid in the core of the jet transits this uptrough sector, the narrower width of the trough relative to the crest. That is, when these shape features are present in the $b(x, y, z)$ field, the frontogenetic tendencies provide positive feedback to further enhance them. This is the primary result of this paper.

These spatial relationships for $\mathscr{T}^{\mathbf{u}}$ and $\mathscr{T}^{b}$ imply a sharpening of the horizontal cross-front buoyancy and velocity gradients in the uptrough sector. The fronts become broader in the downtrough sector where cross-front perturbations and submesoscale instabilities evidently can grow [section $6 \mathrm{~d}(3)$ ], as seen both in observations (Fig. 1) and simulations (e.g., Figs. 4, 7).

We can compare Figs. 17 and 18 based on BE-SCFT solutions with some simpler diagnostic approximations. If, instead of the $\mathrm{BE} \mathbf{u}_{3}$ velocity, a geostrophic $\mathbf{u}_{g}$ horizontal velocity is used in evaluating the frontogenetic tendencies in the right sides of (9), $\mathscr{T}^{\mathbf{u}}$ is zero and $\mathscr{T}^{b}$ has a similar pattern to Fig. 17 but is about $30 \%$ weaker in its peak value. If the full QG velocity $\left(\mathbf{u}_{g}+\mathbf{u}_{\mathrm{ga}}, w_{g}\right)$ is used, then the patterns of both $\mathscr{T}^{\mathbf{u}}$ and $\mathscr{T}^{b}$ are similar to their BE counterparts, but the peak magnitudes are nearly twice as large, as can be expected from the partial cancellations in the finite-Ro corrections in Figs. 10-15. Thus, these non-QG corrections to the frontogenetic tendency are quantitatively important.

\section{b. Shape sensitivities}

The standard shape parameters identified in section 5 and used in the preceding BE-SCFT solution are, at
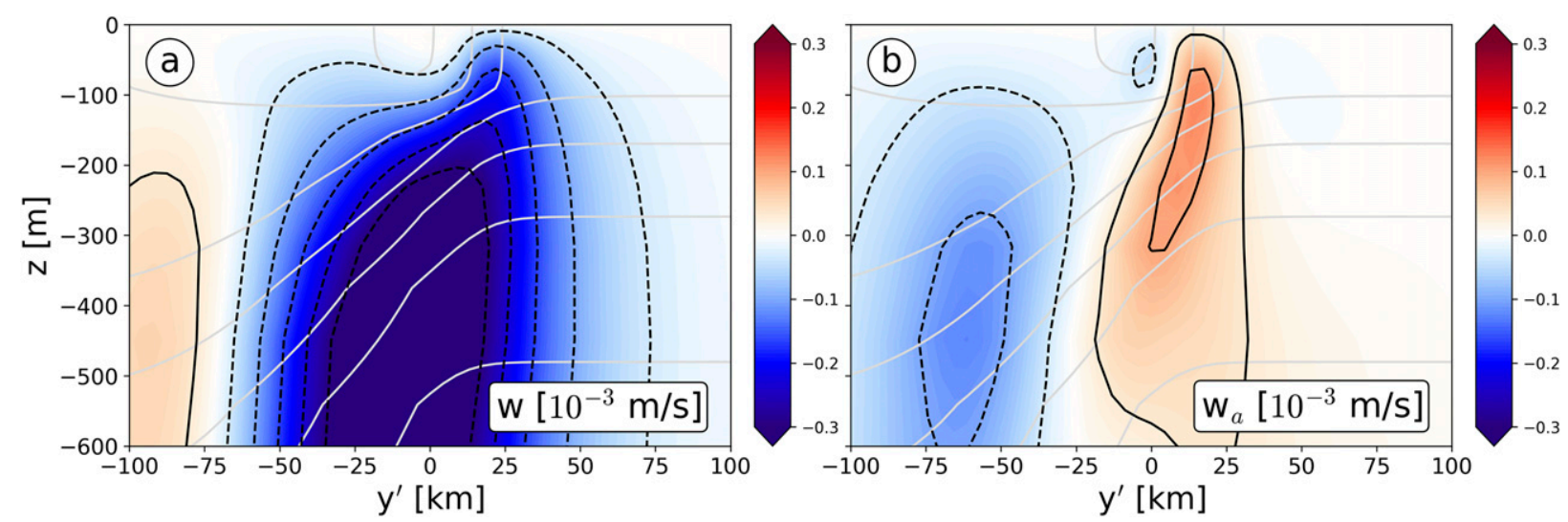

FIG. 15. Vertical velocity $w$ cross section in the middle of the uptrough sector at $x=-100 \mathrm{~km}$ determined from the BE SC analysis of $b$ in Fig. 9 for the standard shape parameters in (13) and (B6): (a) total $w\left(-100, y^{\prime}, z\right)$ and (b) ageostrophic $w_{a}\left(-100, y^{\prime}, z\right)$. The coordinate $y^{\prime}=y=y_{c}(0,0)$ is northward relative to the jet center at the surface in this section. 


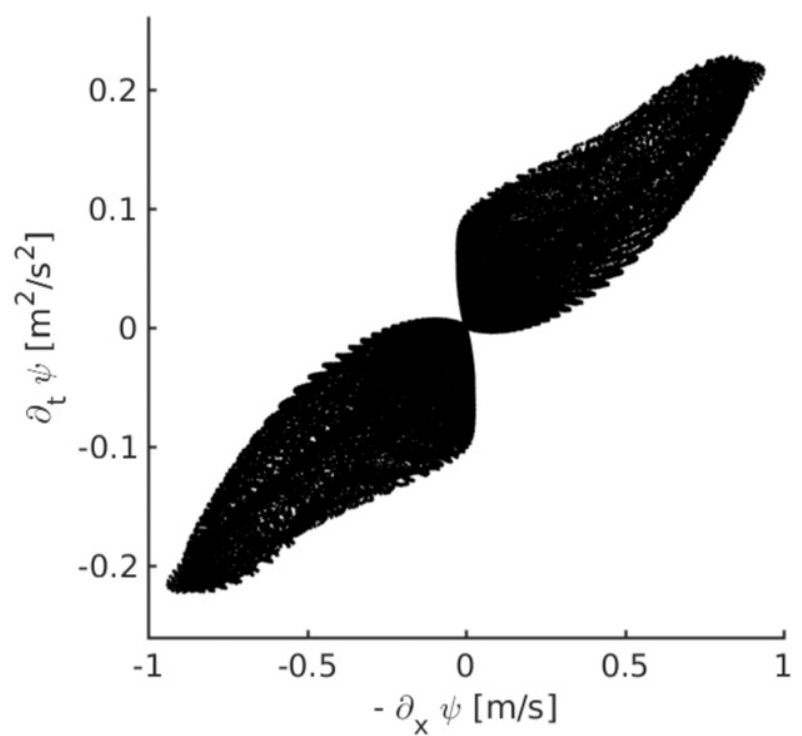

FIG. 16. A scatterplot of $\partial_{t} \psi$ against $-\partial_{x} \psi$ for all grid points for the standard shape parameters in (13) and (B6). The best-fit linear slope to this distribution is the bulk propagation speed of $c$, here approximately equal to $0.22 \mathrm{~m} \mathrm{~s}^{-1}$; see (15) and (16).

best, rough approximations to the more complex shapes that arise in a realistic simulation (section 3). Thus, only a semi-quantitative comparison of this BE-SCFT solution is meaningful. Nevertheless, we can assess how sensitive this solution is to the various choices for the shape parameters to better judge whether it provides a useful interpretation for the simulated Gulf Stream structure.

The simplest dependency is on the horizontal buoyancy anomaly amplitude, that is, $\Delta \bar{b}$ and $b_{*}$ in (13). If these amplitude parameters are rescaled by a factor $q$, then asymptotically in small $q$ the geostrophic fields $\psi_{g}$ and $\zeta_{g}$ change by a factor of $q^{\alpha}$ for $\alpha=1$; the divergent fields $\chi_{g}$ and $w_{g}$ by $\alpha=2$; the ageostrophic $\psi_{a}$ and $\zeta_{a}$ by $\alpha=2 ; \chi_{a}$ and $w_{a}$ by $\alpha=3$; and the frontogenetic tendencies $\mathscr{T}^{b}$ and $\mathscr{T}^{\mathbf{u}}$ by $\alpha=4$. Even for $q=\mathscr{O}(1)$ for the standard BE-SCFT solution in section 6a, these exponents are approximately realized. Thus, in particular, the frontogenetic tendencies are strongly nonlinear functions of the amplitude of the buoyancy anomaly, and only strong jets will exhibit a sharp North Wall.

This amplitude $q$ dependency is equivalent to a Rossby number dependency, at least for small Ro. Thus, BE and QG SCFT solutions differ by $\mathscr{O}(\mathrm{Ro})$. When Ro $=\mathscr{O}(1)$, as here, the corrections to QG are not small, but neither do they completely invalidate the QG estimates.

Another relatively simple dependency is on the meander amplitude parameter $c_{m}$. As described at the start of section 6, the SCFT fields are null for a zonal jet without meandering. For nonzero $c_{m}$ the SCFT dependencies are monotonic increases in amplitude (though not as simple power laws except for small $c_{m}$ ) with relatively little pattern change.

With the full buoyancy anomaly and meander amplitudes, a simple symmetric jet configuration is defined by setting the zonal and meridional asymmetry and tilt parameters to zero in (13) and (B5), $c_{w}=c_{a}=c_{t}=0$. Its SCFT solution is conspicuously different from the standard case, especially with respect to ageostrophic effects. This is illustrated in Fig. 19, which can be compared to Fig. 10. The primary anomaly center in $\psi_{a}$ has shifted from the North Wall in the trough to the south side in the crest, and its amplitude is more than 3 times larger. $\psi_{a}$ exhibits four centers, north and south of the crest and trough, for both jet configurations, consistent with a gradient-wind balance as discussed in association
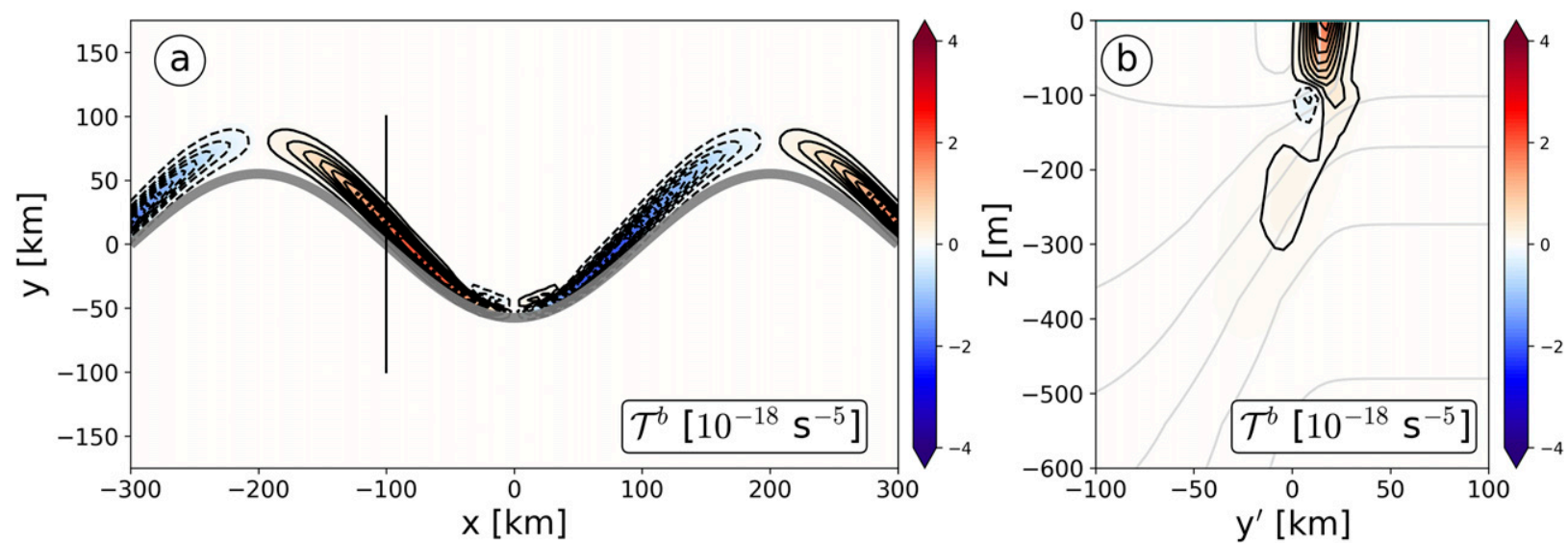

FIG. 17. Frontogenetic tendency for buoyancy $\mathscr{T}^{b}$ in (9), for the idealized $b$ shape in (13) and (B6): (a) surface and (b) a cross section in the middle of the uptrough sector. In (a) the light gray line is the jet center curve at the surface $y_{c}(x, 0)$, and in (b) $y^{\prime}=y-y_{c}(0,0)$ is a northward coordinate relative to the jet center at the surface in this section and the light lines are isopycnals. 

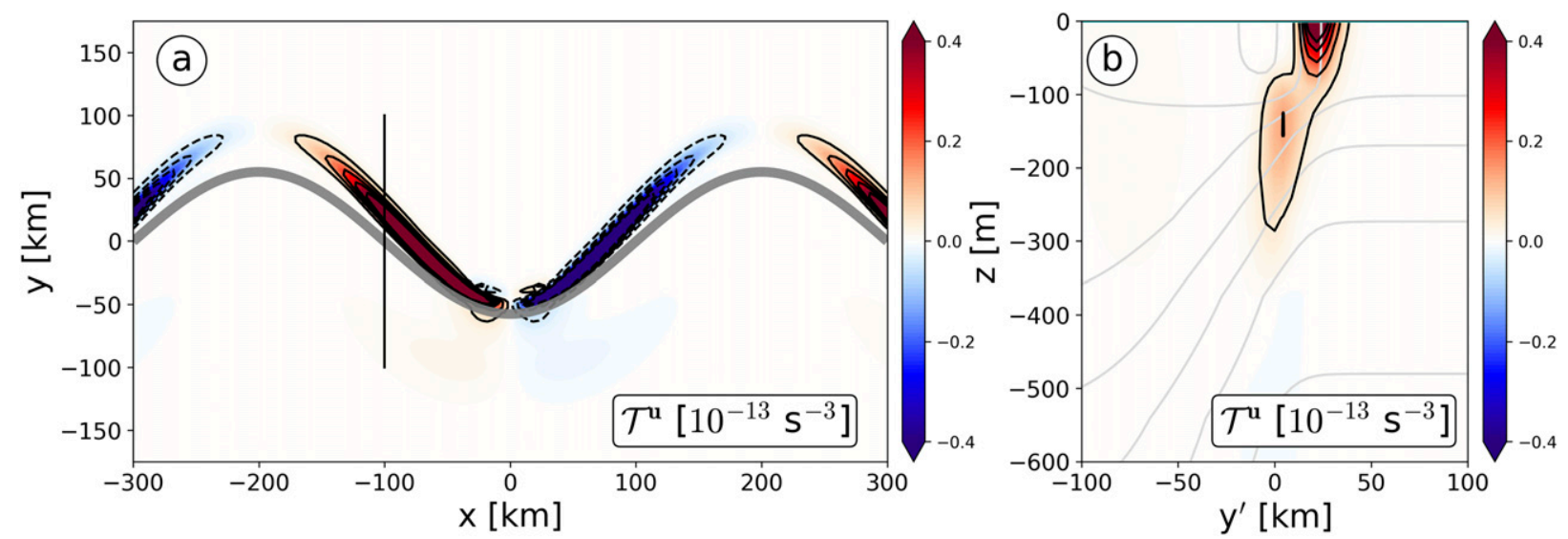

FIG. 18. Frontogenetic tendency for horizontal velocity $\mathscr{T}^{\mathbf{u}}$ in (9), for the idealized $b$ shape in (13) and (B6): (a) surface and (b) a cross section in the middle of the uptrough sector. In (a) the light gray light gray line is the jet center curve at the surface $y_{c}(x, 0)$, and in (b) $y^{\prime}=y-y_{c}(0,0)$ is a northward coordinate relative to the jet center at the surface in this section and the gray lines are isopycnals.

with Fig. 10; however, the shape differences in $b$ between the two configurations change which center is dominant.

The peak amplitudes of both the rotational and divergent fields, including $\zeta$ and $w$, are large and centered around the crest for the simple jet, in contrast to their contiguity to the trough and relatively weaker extrema for the standard jet configuration. The same is true for the frontogenetic tendencies, which no longer show a simple pattern of frontogenesis along the uptrough sector and tilted subsurface front. Thus, a simple jet configuration is not qualitatively consistent with the ROMS patterns shown in section $3 \mathrm{~b}$. The discrepancies between the SCFT fields for the two jet configurations are not as great for their QG solutions as they are for the more complete $\mathrm{BE}$ ones.

The prognostic BE solvability condition $A-S>0$ (section 4) is assessed in the standard and simple-jet
BE-SCFT solutions [also see section 6d(1)]. The $A-S$ tends to have large positive values on the North Wall and smaller ones on the south wall, consistent with the positive and negative signs of $\zeta$, respectively. In the standard case $A-S$ is everywhere positive, even along the south side, whereas it is significantly negative along the south side the simple jet case. This has the implication that a strong meandering jet with Ro $\sim 1$ cannot have a mostly balanced dynamical evolution unless it has at least some of the shape asymmetries identified in section 5. Further, if there is a generic adjustment tendency towards balanced flows in rotating, stratified flows, then any simple symmetric jet configuration would be likely to evolve toward one with more of these shape asymmetries.

By examining other meandering jet configurations with different $c_{w}, c_{a}$, and $c_{t}$ values, we can discern their relative roles in controlling the transitions from the
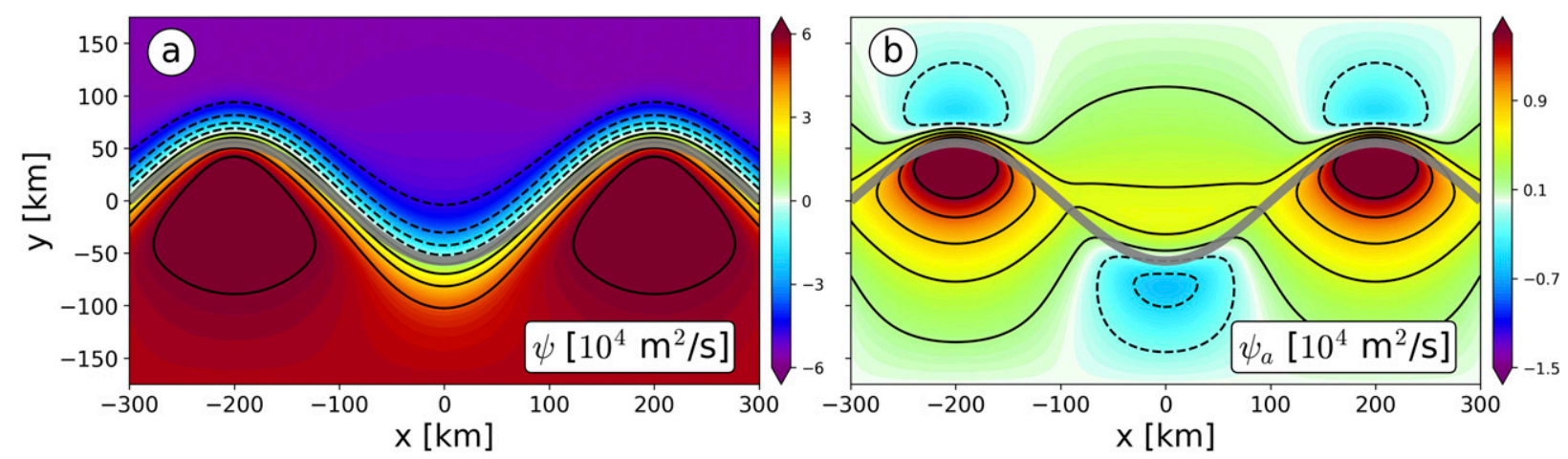

FIG. 19. Streamfunction field $\psi$ at the surface determined from the BE SC analysis of $b$ for the simple symmetric jet configuration with $c_{w}=c_{a}=c_{t}=0$ : (a) total $\psi(x, y, 0)$ and (b) ageostrophic $\psi_{a}(x, y, 0)$. The thin gray line is the jet center at the surface $y_{c}(x, 0)$, which is unchanged by these different parameter values. This can be compared to the ROMS-matched $\psi$ fields in Fig. 10. Note the different amplitude scales in the two plots. 
preceding simple symmetric jet configuration to the standard one in section 6a. Broadly summarizing these alternative SCFT solutions with the same buoyancy amplitude parameters and $c_{m}$ value, nonzero values of both $c_{w}$ and $c_{a}$ are influential in shifting the circulation extrema from the crest to the trough and in concentrating the patterns of downwelling and frontogenetic tendencies on the North Wall of the uptrough sector. ${ }^{3}$ The effect of setting only $c_{t}$ to zero is to strengthen the surface circulation and frontogenetic tendency amplitudes without large changes in their patterns; this is because the hydrostatic integration of a vertically aligned $b$ field yields larger surface pressure and $\eta$ magnitudes.

Finally, the influence of the buoyant jet core can be assessed by setting $b_{*}=0$ with otherwise the standard shape parameter values. This has the effects of modestly weakening the surface rotational circulation, strengthening and broadening the divergent circulation, and weakening the surface frontogenetic tendencies, all without a great change in their respective patterns. Thus, the buoyant core is an amplifier but not an essential cause of the SCFT effects seen in the standard jet solution.

\section{c. SCFT validation}

The realistic simulations in Figs. 4-7 (section 3) exhibit qualitatively similar buoyancy and circulation patterns as shown in the BE-SCFT solutions for the idealized meandering jet in Figs. 9-15.

The frontogenetic tendencies for buoyancy and horizontal velocity (Fig. 20) also exhibit qualitatively similar patterns in the realistic simulations and in the BE-SCFT solutions (Figs. 17, 18). In particular, the frontogenetic tendencies are strongly positive in the uptrough sector and mostly negative in the downtrough sector, albeit with more small-scale fluctuations in the full simulation (Fig. 20). A similar pattern of a positive frontogenetic tendency in the uptrough sectors is seen for the jet stream (Davies and Rossa 1998).

The realistic simulations show a much stronger variability than the idealized solutions, mostly due to the presence of intense submesoscale frontal dynamics. Submesoscale structures stand out in the frontogenetic tendencies, especially in the frontogenetic tendency of

\footnotetext{
${ }^{3}$ As a technical aside, the value of $c_{a}$ chosen for the standard-jet BE-SCFT solutions is somewhat smaller than would be a best fit to the ROMS simulations, as demonstrated by smaller peak cyclonic vorticity in the former case; this is due to a grid-size constraint on the use of MATLAB matrix inversions for the elliptic problems in the BE-SCFT model (section 4). Nevertheless, the qualitative influence of increasing $c_{a}$ is clear from the solutions obtained.
}

velocity. Frontogenetic tendencies in Fig. 20 have been horizontally smoothed to highlight mesoscale patterns. The amplitude of the smoothed $\mathscr{T}^{b}$ is consistent with the $\mathscr{T}^{b}$ in the idealized model, however the amplitude of the smoothed $\mathscr{T}^{\mathbf{u}}$ is still an order of magnitude larger than in the idealized model. The frontogenetic tendencies in a realistic simulation with coarser resolution (the parent simulation with $\delta x=1.5 \mathrm{~km}$ ), where submesoscale dynamics is less prevalent, is much more consistent in its $\mathscr{T}^{\mathbf{u}}$ amplitude with the idealized model. Frontogenetic tendencies for a qualitatively similar meander from the parent simulation have the same pattern and amplitudes than the idealized jet without additional spatial smoothing (Fig. 21). Furthermore, as remarked in section $6 \mathrm{~b}$, the sharpness of the North Wall, controlled by the parameter $c_{a}$, is restricted by the SCFT grid resolution to be as large as to best fit the $\delta x=0.5 \mathrm{~km}$ simulation; hence, some of the more sensitive quantities, like $\mathscr{T}^{\mathbf{u}}$, are somewhat underestimated.

\section{d. Further implications}

The preceding SCFT diagnostic analysis provides only a snapshot of an evolving Gulf Stream meander. A full life cycle would encompass its initiation somewhere after separating from the western boundary and its termination either in spawning a Ring or decaying away farther downstream. In addition, other dynamical processes may emerge beyond those present in the conservative BE solution for the specified $b(\mathbf{x}, z)$ shape, and a few of these are now discussed briefly.

\section{1) LOSS OF BALANCE}

In section 4 several conditions are listed for the solvability of the BE, hence the capability for further evolution consistent with the momentum-balance assumptions. These conditions can be evaluated post hoc for the SCFT-BE solutions in, for example, section $6 \mathrm{a}$. The condition for non-negative stratification, $\partial_{z} b \geq 0$, is assured by design in (13) in section 5 , but it is close to failing in the weakly stratified surface layer; thus, we can expect convective boundary layer turbulence to arise in the presence of surface cooling and a breakdown of momentum balance. The condition for non-negative potential vorticity, $f q \geq 0$, is satisfied below the surface layer, but it fails weakly on the north side of the jet in the surface layer, especially near the trough; thus, we can expect centrifugal or symmetric instability, particularly when downfront winds act to further reduce $f q$ near the surface (Thomas et al. 2013, 2016). Finally, the condition for non-negative $A-S$ is closest to being violated on the south side of the Stream near the trough, extending down into the upper pycnocline; this indicates the flow is close to exhibiting anticyclonic ageostrophic instability. 

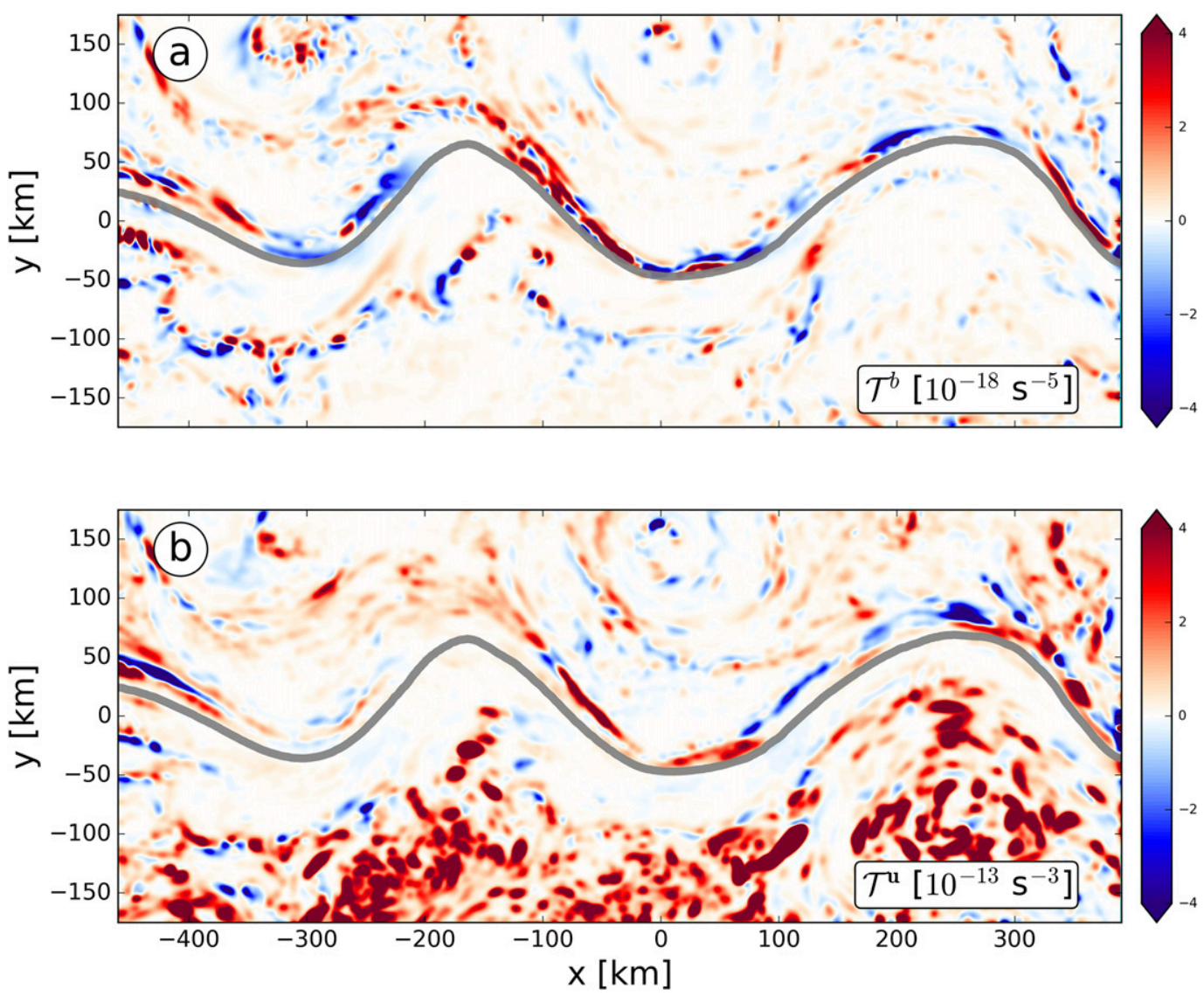

FIG. 20. (a) Frontogenetic tendency of buoyancy $\mathscr{T}^{b}$, and (b) frontogenetic tendency of velocity $\mathscr{T}^{\mathbf{u}}$ at $50-\mathrm{m}$ depth for the simulated Gulf Stream meander of Fig. 4. Frontogenetic tendencies have been smoothed using a convolution with a 20-km half-width Gaussian kernel. The light gray line is the approximate jet center at the surface defined by $\eta=0.3 \mathrm{~m}$.

The marginality of these conditions being satisfied for the idealized meander shape suggests they are likely to be violated on occasion in the real Gulf Stream, and all of the associated instability modes are inconsistent with the momentum-balance assumption. For a weaker jet with smaller Ro, these conditions would be safely satisfied. This suggests the possibility that real meandering jets can persistently be only as strong as the limits of balance allow.

\section{2) BOUNDARY LAYER MIXING AND SURFACE GRAVITY WAVES}

In the surface boundary layer, fronts and filaments exhibit secondary circulations and frontogenesis through the turbulent thermal wind (TTW). The proximate causes are $\nabla b \neq 0$ and vertical momentum mixing with an eddy viscosity $\nu_{v}>0$, as well as Stokes drift from surface gravity waves (McWilliams 2017, 2018). These conditions will occur in the Gulf Stream, and we can expect additional SCFT effects from TTW beyond those shown in the conservative SCFT analysis in section 4. A scaling estimate for the TTW vertical velocity is $w \sim \nu_{v}|\nabla b| / f^{2} l$, which can easily be as large as $10^{-4} \mathrm{~m} \mathrm{~s}^{-1}$ near the Gulf Stream front, that is, comparable to the $w$ magnitudes shown in Figs. 6 and 14. The pattern for $w$ is like that of a surface density front, with downwelling on the dense (north) side, upwelling on the light (south) side, and a vertical extent matching the surface boundary layer depth. There are at least hints of this structure in the simulation's $w$ and $\delta$ fields (Figs. 6a, 7b). The TTW incremental contributions to the frontogenetic tendency are positive along the North Wall, adding to its sharpness near the surface. This is consistent with the stronger amplitudes of positive tendencies in the uptrough sector compared to the negative tendencies in the downtrough sector in the upper mixed-layer for the realistic simulations. The surface divergence pattern in the uptrough sector, where the front is the most intense, has a positive sign south of the front and negative north of the front, which is typical of TTW. The strong positive frontal 

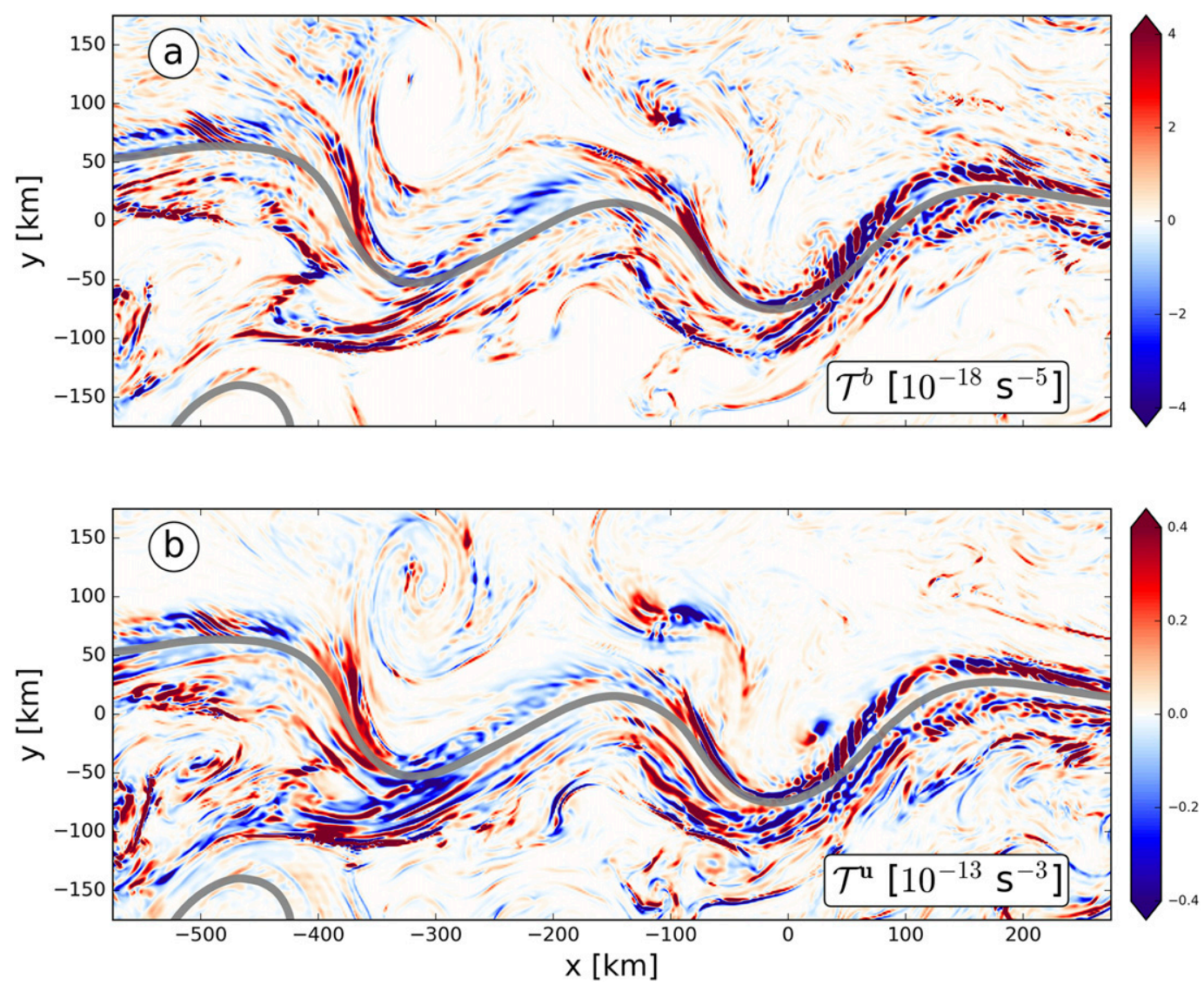

FIG. 21. (a) Frontogenetic tendency of buoyancy $\mathscr{T}^{b}$, and (b) frontogenetic tendency of velocity $\mathscr{T}^{\mathbf{u}}$ at $50-\mathrm{m}$ depth for a meander in the parent-grid simulation with $\delta x=1.5 \mathrm{~km}$. The light gray line is the approximate jet center at the surface defined by $\eta=0.3 \mathrm{~m}$.

tendencies for velocity for the submesoscale fronts and filaments observed, for example, during winter in the region south of the Gulf Stream, are also signatures of TTW frontal sharpening (Callies et al. 2015).

\section{3) Submesoscale instability}

A balanced flow like a meandering jet can manifest instabilities both on the mesoscale (as discussed in section 5) and on the submesoscale. In turn, the latter can either be essentially dynamically balanced, while having a small spatial scale (e.g., the so-called "mixedlayer instability"; Fox-Kemper et al. 2008), or be essentially ageostrophic as in the types mentioned in section $6 \mathrm{~d}(1)$.

One example of an instability in a surface-layer dense filament in the Gulf Stream is in Gula et al. (2014). Other submesoscale instabilities are evident in the solution presented in section 3 (Fig. 7), including one especially prevalent along the North Wall near the surface in the downtrough and crest sectors (to be reported on later). Thus, the Gulf Stream has active submesoscale currents beyond the idealized meander structure examined in this paper, and their characteristic behaviors are distinct from more open-ocean submesoscale flows.

\section{Summary and discussion}

This paper demonstrates with a momentum-balanced diagnostic analysis how various aspects of the shape of the offshore Gulf Stream's buoyancy field control its ageostrophic secondary circulation and reinforce a sharp front in the horizontal gradients of both buoyancy and velocity at its northern side (the Wall), especially along the meander sector upstream from and extending through the trough. The frontogenesis effecting the latter appears to have the further effect of suppressing submesoscale activity along this sector and near its termination at the meander trough, though this relationship has yet to be fully explained. These patterns and behaviors are evident also in both measurements and realistic simulations. 
These shapes and their consequences are hypothesized to be generic for meandering eastward jets in the atmosphere and ocean at the height of their maximum velocity, with similar patterns in their buoyancy and velocity fields, and with frontogenesis and fluctuation suppression on the polar side of their uptrough meander sectors. This implies a positive feedback relationship: narrowness in the polar side and in the troughs, and an equatorward tilt with depth, all beget further frontogenetic narrowing.

The relevance of this analysis to the atmospheric jet streams is only slightly developed in this paper. While the assumed shape attributes (section 5) are demonstrably apt (Keyser and Shapiro 1986), there are important phenomenological differences with the Gulf Stream as well. The oceanic idealization here is as a zonally periodic meander that propagates eastward with a slow temporal evolution in its shape, although of course exceptions occur with aperiodic patterns and dramatic disruptions like a ring pinchoff. The more common atmospheric idealization is closely tied to the "baroclinic life cycle" of midlatitude cyclones, which both implies a more active temporal evolution and strong coupling between the upper-level jet and the lower-level storm vortex that has no oceanic counterpart. Thus, quite a different interpretive language has developed for the atmosphere that inhibits facile comparisons of dynamical similarity and difference. Nevertheless, a reading of the literature supports strong tropopause secondary circulation and frontogenesis in the uptrough region (Shapiro 1981; Shapiro and Keyser 1990; Rotunno et al. 1994; Keyser 1999; Bosart 2003), sometimes referred to as the "Shapiro effect." A more thorough consideration of this interesting and important topic is worth pursuing.

This demonstration is only a partial theoretical explanation of this phenomenon. As a diagnostic secondary circulation and frontogenetic tendency (SCFT) analysis, it only applies to an instantaneous realization of an evolving pattern. What gives it some generality is the experimental fact that the meander pattern analyzed here seems to be typical of a recurrent (coherent) spatial pattern within a characteristic life cycle of the continuous evolution in eastward jets. Future work might attempt a more complex characterization of a meander life cycle than presented here.

It is also unclear from the present simulation and SCFT analyses what the net frontogenetic tendency is when integrated over a whole meander wavelength and combining the opposing tendencies in the uptrough and downtrough sectors. Figure 1 suggests the North Wall can be sharp even at the point of western-boundary separation, so there may still be more to explain about North Wall sharpness. Surface-layer TTW frontogenesis [section $6 \mathrm{~d}(2)]$ is persistent along the North Wall for the entire meander length and may be part of the explanation. However, we can further speculate that it is likely that there is net frontogenesis by the following argument. The idealized $b$ shape in section 5 is symmetric in the zonal direction about both troughs and crests, leading to antisymmetric frontogenetic tendencies (Figs. 17, 18). However, the positive frontogenesis occurring in the uptrough sector will lead to a sharper north wall and narrower jet there and in the trough, with vice versa in the downtrough sector and crest. This asymmetry between the faces probably will imply stronger uptrough frontogenesis than downtrough frontolysis, that is, a net north wall frontogenesis over a full meander length. (We did not incorporate the additional complexity of this type of asymmetry in our $b$ shape to test this expectation.)

The specific idealizations of the Gulf Stream shape in section 5 are somewhat arbitrary, as are the choices of the shape parameter values in relation to any particular realization of a single meander period in the turbulent and chaotic evolution of the flow. Nevertheless, there is qualitative agreement between the observed and simulated patterns, on the one hand, and the idealized patterns on the other.

The particular choice here of a momentum-balanced approximation based on Lorenz (1960) is also nonunique relative to the many different ideas previously proposed for rotating, stratified flows. However, its solutions here, as in previous studies, do seem apt by comparison with more general model solutions (e.g., a ROMS simulation). These SCFT solutions are conservative ones that do not include the effects of surface forcing and interior mixing. They do show that in a strong jet like the Gulf Stream with its large Ro values, there are qualitatively and quantitatively important influences compared to the simpler (small Ro) approximate model of quasigeostrophy. The more general simulation also shows active submesoscale processes within the Gulf Stream that are not present in the spatially smooth, idealized shapes assumed in the SCFT analysis. The aptness of the SCFT solutions indicates that this evident mesoscale-submesoscale interaction is not a crucial element in the shape and dynamics of the meanders themselves.

The Gulf Stream is thus significantly ageostrophic in its dynamical evolution, even if some simple features such as the existence of meanders, zonal propagation, and broad pattern of vertical velocity are qualitatively consistent with a QG model. The detailed structure of its 3D shape, secondary circulation, frontogenetic 
tendency, and instabilities (not analyzed here) are partly ageostrophic but mostly "balanced." By implication the same statements are probably true for other strong jets in the ocean and atmosphere.

Acknowledgments. We appreciate conversations with Mel Shapiro, Lance Bosart, and Gang Chen about the atmospheric jet stream. This research was supported by the Office of Naval Research through Grants N000141210939 and N000141410626 and by LEFE/IMAGO through the Project AO2017-994457RADII. Some of the computations were made at the San Diego Supercomputing Center as part of the
Extreme Science and Engineering Discovery Environment (XSEDE) supported by the National Science Foundation. ROMS simulations were performed using HPC resources from GENCI-TGCC (Grant 2017A0010107638) and using the HPC facilities DATARMOR of "Pôle de Calcul Intensif pour la Mer" at Ifremer, Brest, France.

\section{APPENDIX A}

\section{Fit to Far-Field Buoyancy Profiles}

The shape functions for $\bar{b}_{S}(z)$ and $\bar{b}_{N}(z)$ are defined by

$$
\bar{b}_{\bullet}(z)= \begin{cases}b_{0}+b_{\bullet 0}+N_{0}^{2}(z+H), & \text { if } \quad \hat{z} \leq 0, \\ b_{0}+b_{\bullet \bullet} \frac{1-\left[\hat{z}_{\bullet} /\left(H-h_{\bullet \bullet}\right)\right]^{n}}{1+\left[\hat{z}_{\bullet} /\left(h_{\bullet p}-h_{\bullet s}\right)\right]^{n}}+N_{0}^{2}(z+H) & \text { if } \quad \hat{z} \geq 0\end{cases}
$$

where $\hat{z}=-\left(z+h_{\bullet s}\right)$ and $\bullet=S, N$. There is no physically specific meaning to this functional form; it is merely a means of representing the stratification profiles on either side of the Gulf Stream. The multiple parameters are fit to area averages of the ROMS solution (section 3) on either side of the Stream, and the results are plotted in Fig. 8 for the following parameter values:

$$
\begin{aligned}
& b_{S 0}=18.7 \times 10^{-3} \mathrm{~m} \mathrm{~s}^{-2}, \quad h_{S s}=130 \mathrm{~m}, \quad h_{S p}=850 \mathrm{~m}, \quad n_{S}=2.0 \text {, } \\
& b_{N 0}=13.6 \times 10^{-3} \mathrm{~m} \mathrm{~s}^{-2}, \quad h_{N s}=90 \mathrm{~m}, \quad h_{N p}=340 \mathrm{~m}, \quad n_{N}=1.05 \text {, }
\end{aligned}
$$

plus $b_{0}=0, N_{0}^{2}=10^{-8} \mathrm{~s}^{-2}$, and $H=1500 \mathrm{~m}$. This functional form for the fit has two vertical length scales, one associated with the weakly stratified surface layer $h_{*}$ and another associated with the pycnocline depth $h_{*_{p}}$, where the decay exponent $n_{*}$ is chosen to match the shapes. Parameter $b *_{0}$ represents the overall buoyancy increment in the interval $[-H, 0]$. The parameter $N_{0}^{2}$ guarantees a minimum level of stable stratification even in the surface layer [as required for a wellbehaved elliptic operator in $\Omega E$ in (8)].

\section{APPENDIX B}

\section{Functional Form for $b(x, y, z)$}

For the idealized $b(x, y, z)$ shape in (13), the jetcenter location is

$y_{c}(x, z)=-l_{0}\left\{c_{m} \cos \left[\frac{2 \pi x}{L_{x}}\right]+c_{t} \mathscr{F}(z) \xi(x)\right\}$.
Parameter $l_{0}$ is the basic half-width scale for the jet. The first term is the meandering jet-center position at the surface with a nondimensional amplitude $c_{m}$. The zonal location $x=0$ corresponds to a trough $\left[y_{c}(x, 0)<0\right]$, and $x= \pm L_{x} / 2$ corresponds to crests, with $L_{x}$ the zonal periodicity length. The second term represents the southward tilt of the jet center with depth, relative to the surface meander position. The function

$$
\xi(x)=1-c_{w} \cos \left[\frac{2 \pi x}{L_{x}}\right], \quad c_{w} \geq 0,
$$

is a nondimensional modulation of the width of the jet along its meandering axis with a relative amplitude of $c_{w}: \xi$ is a minimum at a trough and maximum at a crest. Parameter $c_{t}$ is its nondimensional tilt distance relative to $l_{0}$. The vertical function

$$
\mathscr{F}(z)=\left\{\begin{array}{ccc}
0, & \text { if } & z \geq-h_{s}, \\
\hat{\zeta}^{2} /\left(0.25+\hat{\zeta}^{2}\right), & \text { if } & z<-h_{s},
\end{array}\right.
$$


is the vertical profile of the jet-center tilt, with

$$
\hat{\zeta}(z)=-\frac{z+h_{s}}{h_{p}-h_{s}}
$$

a rescaled vertical coordinate near the surface. There is no tilt within the surface layer, it increases with depth on the scale of $h_{p}$, and it asymptotes at depth to $\mathscr{F}=1$. Because the tilt is consequential only near the jet itself, we chose $h_{s}$ and $h_{p}$ in the middle as the averages of their $S, N$ counterparts in (A2), that is, 110 and $595 \mathrm{~m}$, respectively.

The jet width is defined by

$$
l(x, y, z)=l_{0} \xi(x)\left\{1+\frac{c_{a}}{2} \tanh \left[\frac{y_{c}(x, z)-y}{c_{a} l_{0} \xi(x)}\right]\right\} .
$$

It varies with zonal position along the jet as specified in (B2), and it also is narrower on the northern side $\left(y>y_{c}\right)$ to a degree controlled by the nondimensional, cross-jet profile asymmetry amplitude $c_{a}>0$.

With the formula (13) and all constants $c_{\bullet}=0$, the jet is a straight zonal flow, uniform in $x$, and meridionally symmetric. Nonzero values of the $c_{\bullet}$ represent the structural features of meandering $c_{m}$, width modulation $c_{w}$, North Wall sharpness $c_{a}$, and vertical tilt $c_{t}$. We chose the reference half-width of the jet as $l_{0}=30 \mathrm{~km}$. $b_{*}=6.0 \times 10^{-3} \mathrm{~m} \mathrm{~s}^{-2}$ is the surface magnitude of the buoyant central core, relative to $\Delta \bar{b}(0)=5.1 \times 10^{-3} \mathrm{~m} \mathrm{~s}^{-2}$. The depth scale for the buoyant core is $h_{*}=30 \mathrm{~m}$. For the jet shape parameters $c_{\bullet}$, we choose

$$
c_{m}=2.0, \quad c_{w}=0.4, \quad c_{a}=1.0, \quad c_{t}=2.0 .
$$

\section{REFERENCES}

Bosart, L. F., 2003: Topopause folding, upper-level frontogenesis, and beyond. A Half-Century of Progress in Meteorology: A Tribute to Richard Reed, R. H. Johnson and R. A. Houze, Eds., Amer. Met. Soc., 13-47.

Bower, A., 1989: Potential vorticity balances and horizontal divergence along particle trajectories in Gulf Stream meanders east of Cape Hatteras. J. Phys. Oceanogr., 19, 1669-1681, https://doi.org/10.1175/1520-0485(1989)019<1669:PVBAHD> 2.0.CO;2.

_ 1991: A simple kinematic mechanism for mixing fluid parcels across a meandering jet. J. Phys. Oceanogr., 21, 173-180, https://doi.org/10.1175/1520-0485(1991)021<0173:ASKMFM> 2.0.CO;2.

Callies, J., R. Ferrari, J. M. Klymak, and J. Gula, 2015: Seasonality in submesoscale turbulence. Nat. Commun., 6, 6862-6870, https://doi.org/10.1038/ncomms7862.

Chekroun, M. D., H. Liu, and J. C. McWilliams, 2017: The emergence of fast oscillations in a reduced primitive equation model and its implications for closure theories. Comput. Fluids, 151, 3-22, https://doi.org/10.1016/j.compfluid.2016.07.005.
Davies, H. C., and A. M. Rossa, 1998: PV frontogenesis and upper-tropospheric fronts. Mon. Wea. Rev., 126, 1528-1539, https:// doi.org/10.1175/1520-0493(1998)126<1528:PFAUTF>2.0.CO;2.

Ducet, N., P.-Y. Le Traon, and G. Reverdin, 2000: Global highresolution mapping of ocean circulation from TOPEX/Poseidon and ERS-1 and-2. J. Geophys. Res., 105, 19477-19498, https:// doi.org/10.1029/2000JC900063.

Fox-Kemper, B., R. Ferrari, and R. W. Hallberg, 2008: Parameterization of mixed layer eddies. Part I: Theory and diagnosis. J. Phys. Oceanogr., 38, 1145-1165, https://doi.org/10.1175/ 2007JPO3792.1.

Gula, J., M. J. Molemaker, and J. C. McWilliams, 2014: Submesoscale cold filaments in the Gulf Stream. J. Phys. Oceanogr., 44, 2617-2643, https://doi.org/10.1175/JPO-D-14-0029.1.

,$- \ldots$, and $—, 2015$ : Gulf Stream dynamics and frontal eddies along the southeastern U.S. seaboard. J. Phys. Oceanogr., 45, 690-715, https://doi.org/10.1175/JPO-D-14-0154.1.

Hoskins, B. J., and F. P. Bretherton, 1972: Atmospheric frontogenesis models: Mathematical formulation and solution. J. Atmos. Sci., 29, 11-37, https://doi.org/10.1175/1520-0469(1972)029<0011: AFMMFA $>2.0 . \mathrm{CO} ; 2$.

Keyser, D., 1999: On the representation and diagnosis of frontal circulations in two and three dimensions. The Life Cycles of Extratropical Cyclones, C. Newton and S. Gronas, Eds., Amer. Met. Soc., 239-264.

— namics of upper-level frontal zones. Mon. Wea. Rev., 114, 452-499, https://doi.org/10.1175/1520-0493(1986)114<0452: AROTSA $>2.0 . \mathrm{CO} ; 2$.

Klymak, J., and Coauthors, 2016: Submesoscale streamers exchange water on the North Wall of the Gulf Stream. Geophys. Res. Lett., 43, 1226-1233, https://doi.org/10.1002/2015GL067152.

Lindstrom, S., X. Qian, and D. Watts, 1997: Vertical motion in the Gulf Stream and its relation to meanders. J. Geophys. Res., 102, 8485-8503, https://doi.org/10.1029/96JC03498.

Lorenz, E., 1960: Energy and numerical weather prediction. Tellus, 12, 364-373, https://doi.org/10.3402/tellusa.v12i4.9420.

McWilliams, J. C., 2017: Submesoscale surface fronts and filaments: Secondary circulation, buoyancy flux, and frontogenesis. J. Fluid Mech., 823, 391-432, https://doi.org/10.1017/ jfm.2017.294.

- 2018: Surface wave effects on submesoscale fronts and filaments. J. Fluid Mech., 843, 479-817, https://doi.org/10.1017/ jfm.2018.158.

- and P. R. Gent, 1980: Intermediate models of planetary circulations in the atmosphere and ocean. J. Atmos. Sci., 37, 1657-1678, https://doi.org/10.1175/1520-0469(1980)037<1657: IMOPCI $>2.0 . \mathrm{CO} ; 2$.

—, I. Yavneh, M. J. P. Cullen, and P. R. Gent, 1998: The breakdown of large-scale flows in rotating, stratified fluids. Phys. Fluids, 10, 3178-3184, https://doi.org/10.1063/1.869844.

_ L. P. Graves, and M. T. Montgomery, 2003: A formal theory for vortex Rossby waves and vortex evolution. Geophys. Astrophys. Fluid Dyn., 97, 275-309, https://doi.org/10.1080/ 0309192031000108698.

Nagai, T., A. Tandon, H. Yamazaki, and M. Doubell, 2009: Evidence of enhanced turbulent dissipation in the frontogenetic Kuroshio Front thermocline. Geophys. Res. Lett., 36, L12609, https://doi.org/10.1029/2009GL038832.

Rotunno, R., C. Snyder, and W. Skamarock, 1994: An analysis of frontogenesis in numerical simulations of baroclinic waves. J. Atmos. Sci., 51, 3373-3398, https://doi.org/10.1175/ 1520-0469(1994)051<3373:AAOFIN>2.0.CO;2. 
Schubert, R., A. Biastoch, M. Cronin, and R. Greatbatch, 2018: Instability-driven benthic storms below the separated Gulf Stream and the North Atlantic Current in a high-resolution ocean model. J. Phys. Oceanogr., 48, 2283-2303, https:// doi.org/10.1175/JPO-D-17-0261.1.

Shapiro, M. A., 1981: Frontogenesis and geostrophically forced secondary circulations in the vicinity of jet stream-frontal zone systems. J. Atmos. Sci., 38, 954-972, https://doi.org/10.1175/ 1520-0469(1981)038<0954:FAGFSC >2.0.CO;2.

- and D. Keyser, 1990: Fronts, jet streams, and the tropopause. Extratropical Cyclones: The Erik Palmén Memorial Volume, C. Newton and E. O. Holopainen, Eds., Amer. Met. Soc., 167-189.

Shchepetkin, A. F., and J. C. McWilliams, 2005: The Regional Oceanic Modeling System (ROMS): A split-explicit, freesurface, topography-following-coordinate oceanic model. Ocean Modell., 9, 347-404, https://doi.org/10.1016/j.ocemod. 2004.08.002.

Thomas, L. N., and T. Joyce, 2010: Subduction on the northern and southern flanks of the Gulf Stream. J. Phys. Oceanogr., 40, 429-438, https://doi.org/10.1175/2009JPO4187.1.
__ J. R. Taylor, R. Ferrari, and T. Joyce, 2013: Symmetric instability in the Gulf Stream. Deep-Sea Res. II, 91, 96-110, https://doi.org/10.1016/j.dsr2.2013.02.025.

——, E. A. D'Asaro, C. M. Lee, J. M. Klymak, and A. Shcherbina, 2016: Symmetric instability, inertial oscillations, and turbulence at the Gulf Stream front. J. Phys. Oceanogr., 46, 197-217, https://doi.org/10.1175/JPO-D-15-0008.1.

Todd, R., and B. Owens, 2016: Gliders in the Gulf Stream. Scripps Institution of Oceanography, Instrument Development Group, https://doi.org/10.21238/s8SPRAY2675.

Todd, R. E., W. B. Owens, and D. L. Rudnick, 2016: Potential vorticity structure in the North Atlantic western boundary current from underwater glider observations. J. Phys. Oceanogr., 46, 327-348, https://doi.org/10.1175/JPO-D15-0112.1.

Yavneh, I., A. F. Shchepetkin, J. C. McWilliams, and L. P. Graves, 1997: Multigrid solution of rotating, stably stratified flows: The balance equations and their turbulent dynamics. J. Comput. Phys., 136, 245-262, https://doi.org/10.1006/ jcph.1997.5775. 